\title{
Equilibrium Selection in Similar Repeated Games: Experimental Evidence on the Role of Precedents*
}

\author{
John Duffy ${ }^{\dagger}$ and Dietmar Fehr ${ }^{\ddagger}$
}

December 17, 2014

\begin{abstract}
We report on an experiment examining behavior and equilibrium selection in two similar, infinitely repeated games, Stag Hunt and Prisoner's Dilemma under anonymous random matching. We are interested in the role that precedents may play for equilibrium selection between these two stage game forms. We find that a precedent for efficient play in the repeated Stag Hunt game does not carry over to the repeated Prisoner's Dilemma game despite the possibility of efficient play in the latter game. Similarly, a precedent of inefficient play in the Prisoner's Dilemma game does not extend to the repeated Stag Hunt game. We conclude that equilibrium selection between similar repeated games has little to do with historical precedents and is mainly determined by strategic considerations associated with the different payoffs of these similar repeated games.
\end{abstract}

Keywords: Equilibrium Selection, Precedent, Beliefs, Stag Hunt, Prisoner's Dilemma, Repeated Games, Experimental Economics.

JEL classification numbers: C72, C73, C92, D83

${ }^{*}$ Dietmar Fehr gratefully acknowledges financial support from the Deutsche Forschungsgemeinschaft (DFG) through CRC 1029.

${ }^{\dagger}$ University of California, Irvine, USA. Email: duffy@uci.edu

${ }^{\ddagger}$ WZB Berlin, Germany. Email: dietmar.fehr@wzb.eu. 


\section{Introduction}

One of the most important uses of the experimental economics methodology is to address questions of equilibrium selection in environments with multiple equilibria. As Lucas (1986) observed, on the question of equilibrium selection:

"Economic theory does not resolve the question. One can imagine other principles that would, but this cannot rule out the possibility that other principles might resolve it quite differently. It is hard to see what can advance the discussion short of assembling a collection of people, putting them in a situation of interest, and observing what they do."

Numerous experimental studies have explored questions of equilibrium selection in both static and repeated (dynamic) games (for surveys see, e.g., Ochs, 1995 or Camerer, 2003, Chapter 7). The question that we explore in this paper is whether equilibrium selection in one repeated game involving multiple equilibria serves as a precedent for equilibrium selection in a similar repeated game possessing a similar multiplicity of equilibria. That is, we are interested in whether there is a transfer of precedent to new, but similar strategic settings. This question is of real-world importance as it is likely that the payoffs that players face in repeated strategic settings change over time and thus it is of interest to understand whether and how players adapt their behavior to such changes. It is also of interest to understand mechanisms that might support the play of efficient equilibria in repeated game environments where other equilibria are possible, and precedent (or history) is one mechanism that is underexplored in the literature. Our paper takes a first step toward addressing this question by combining the theory of repeated games with experimental economics methods.

The importance of precedence or "history" in coordination games was first established by Van Huyck, Battalio and Beil (1991). They studied behavior in an average opinion game where $n$ players were asked to choose from among the set of integers 1-7 with the common payoff function decreasing in the distance to the median of all numbers chosen. Thus, there are 7 pure Nash equilibria, and Van Huyck et al. found that the median number selected in repeated play was equal to the median number chosen in the very first period which served as a precedent. Rankin, Van Huyck, and Battalio (2000) studied play of a sequence of similar Stag Hunt games that differed in the payoffs and action labels each period. They report that payoff dominance rather than risk dominance was the most frequent selection criterion adopted by subjects. Devetag (2005) documented that efficient play in a critical-mass game transfered in most cases to the Pareto-efficient equilibrium in a seven-choice minimum-effort game. Cason, Savikhin and Sheremeta (2012) provide evidence that play of the Paretooptimal equilibrium in a medium-effort game influences play in a subsequent minimum-effort game. ${ }^{1}$ In work more related to this paper, Knez and Camerer (2000) found that a precedent

\footnotetext{
${ }^{1}$ Other, related papers explore how the salience of equilibrium payoffs (Brandts and Cooper, 2006), reputation building (e.g., Ahn et al, 2001; Bohnet and Huck, 2004; Duffy and Ochs 2009), gradually increasing the group size (Knez, 1998; Weber, 2006) or task difficulty (Dufwenberg, Sundaram and Butler 2010) affect behavior in the same, consecutively played game.
} 
of efficient play in a seven choice minimum-effort coordination game increased cooperation in a subsequent, finitely-repeated seven choice prisoners' dilemma game. Peysakhovich and Rand (2013) report on an experiment where, in a first stage, subjects are anonymously and randomly matched to play an indefinitely repeated prisoner's dilemma game that is parameterized to facilitate adoption of either a social norm of cooperation or a social norm of defection. In a subsequent second stage, subjects are anonymously matched to play a number of different one-shot games, e.g., a public goods game, an ultimatum game, a dictator game and a trust game. They report that subjects who experienced a social norm of cooperative play in stage 1 are more likely to play pro-socially in the subsequent stage 2 one-shot games as compared with subjects who experienced a social norm of defection in stage 1.

Our first point of departure from this literature is that we examine the role of history or precedents in equilibrium selection across similar indefinitely repeated games which serve as proxies for infinitely repeated games. As noted above, the literature has focused on the role of precedents for one-shot or finitely repeated games. However, many real-world strategic interactions are not one-shot encounters or finitely repeated games; it is frequently the case that strategic interactions are indefinitely rather than finitely repeated. Furthermore, in the finitely repeated Prisoner's Dilemma game studied, for example by Knez and Camerer (2000), or in the one-shot games studied by Peysakhovich and Rand (2013) there is no theoretical equilibrium selection question as "defection" by all players is a dominant strategy in the second stage game(s) that they considered.

A second point of departure of our work from the literature is that we are more precise about the similarity of the two repeated games we study enabling us to finely tune the degree of similarity between these two games and examine whether variations in the similarity of the games matters for the role of precedents. In particular, we consider indefinitely repeated play of versions of a $2 \times 2$ stage game - a Prisoner's Dilemma game or a Stag Hunt game. The stage games $\Gamma[T]$ differ only in the "off-diagonal" temptation payoff, $T$, as in the payoff matrix shown in Table 1.

\begin{tabular}{ccc} 
& $\boldsymbol{X}$ & $\boldsymbol{Y}$ \\
\hline $\boldsymbol{X}$ & 20,20 & $0, \mathrm{~T}$ \\
$\boldsymbol{Y}$ & $\mathrm{T}, 0$ & 10,10
\end{tabular}

Table 1: The $2 \times 2$ stage game used in the experiment

All other payoff values in the game are held constant across our experiment; that is, treatments consist only of variations in $T$ or in the order of the games played. For the stage game with $T>20$, the game becomes a Prisoner's Dilemma game as $Y$ (equivalent to defection) is a dominant strategy for both players in the one-shot game. When $10 \leq T<20$ the stage game is a Stag Hunt coordination game with Pareto rankable equilibria as in Van Huyck, Battalio and Beil (1991) with two pure strategy equilibria, both players play $X$ - the cooperative and efficient equilibrium - or both players play $Y$. There is also a mixed strategy 
equilibrium where $X$ is played with probability $\frac{10}{30-T}$.

We study play of this stage game for various values of $T$ under anonymous random matching and indefinite repetition. This is a stark though empirically plausible environment that has been the subject of much study with regard to mechanisms for sustaining social norms of cooperation among strangers. Here the mechanism we consider is historical precedent. The fact that the game is indefinitely repeated means that there can be multiple equilibria in the Prisoner's Dilemma version of the game even under the random anonymous matching protocol that we adopt under certain parameterizations of the environment. ${ }^{2}$ In particular, given the parameterizations of the PD versions of game that we study and the continuation probability for indefinite play, it is possible to support play of the most efficient equilibrium where all players play $X$ via the social norm, contagious strategy of Kandori (1992); the specific details are provided in Appendix A. Prior research, for example, by Duffy and Ochs (2009) suggests that coordination on play of this efficient equilibrium when the stage game is a PD is not easily achieved under anonymous random matching in large populations of size 6 or 14, though it may have some success in smaller populations of size 4, as in Camera and Casari (2009). Here, we consider a population of size 10 and ask whether coordination on a (pure) equilibrium in the repeated Stag Hunt game (e.g., the cooperative outcome where all play $X$ ) or the inefficient outcome where all play $Y$ ) serves as precedent for equilibrium selection in the repeated Prisoner's Dilemma game for the same population of 10 players under anonymous random matching, and we also explore the reverse order of whether a precedent for equilibrium selection in the repeated Prisoner's Dilemma game carries over to the Stag Hunt game. Note that unlike Knez and Camerer (2000) we are not changing the number of actions that players have available between the two games. Furthermore, the two pure strategy equilibria of these repeated games - all play $X$ or all play $Y$ are the same and involve the same payoffs; we are only changing the off-diagonal temptation payoff, $T$.

Our main finding is that the role of precedent for equilibrium selection in the repeated games we study is surprisingly limited. For small changes in the value of the temptation payoff parameter, we observe large swings in the frequency with which players play the two actions available to them. More precisely, the frequency of play of the cooperative action, $X$, is stochastically larger in Stag-hunt game parameterizations of the stage game than in Prisoner's Dilemma parameterizations of the stage game, regardless of the order in which these two types of games are played. These swings in the frequency of cooperative play are associated with significant changes in beliefs, which are largely influenced by players' initial propensities to cooperate as well as the frequency of cooperative encounters early on.

There is, however, some variation in the frequency of play of action $X$ and beliefs about cooperation as the temptation parameter is varied, and there are some instances where a precedent of coordination on the inefficient, all play $Y$ equilibrium in the repeated Prisoner's Dilemma game carries over to the subsequent Stag Hunt game or the reverse (i.e., a precedent of inefficient all $Y$ play in the repeated Stag Hunt game carries over to the subsequent Prisoner's Dilemma game) though such transfer of precedent is not the norm. Importantly,

\footnotetext{
${ }^{2}$ For experimental evidence on cooperation in indefinitely repeated Prisoner's Dilemma games under random matching, see e.g., Duffy and Ochs, 2009; Camera and Casari, 2009 or Dal Bò and Frechette, 2011.
} 
we never observe that a precedent of efficient play, where all play $X$ (as is often achieved in the repeated Stag hunt game), carries over to the subsequent Prisoner's Dilemma game. We conclude that equilibrium selection results for indefinitely repeated games may be rather limited in scope to particulars of the stage game being played and that there appears to be limited use of equilibrium selection precedents between similar indefinitely repeated games.

The rest of the paper is organized as follows. The next section presents our experimental design and procedures and outlines our main hypotheses. Section 3 presents the aggregate level results and section 4 looks at the results at the finer, individual level and tries to explain the observed patterns. Finally section 5 offers conclusions and directions for future research.

\section{Experiment and Hypotheses}

\subsection{Design}

Our experiment makes use of the game $\Gamma[T]$ shown in Table 1 . We vary only the off-diagonal payoff parameter, $T=\{10,15,25,30\}$ to create variation in the similarity of the stage game. More specifically, in each experimental session we consider just two different values, $T$ and $T^{\prime}$, and we use the difference $\left|T-T^{\prime}\right|$ as a measure of the similarity of the two stage games, $\Gamma[T]$ and $\Gamma\left[T^{\prime}\right]$. One pair of parameter values for the stage games consists of $T=10$ and $T=30$ and the other pair consists of $T=15$ and $T=25$. That is, for the stage-game pair $\Gamma[15]$ and $\Gamma[25]$ the difference $\left|T-T^{\prime}\right|=10$ and thus they are more similar than the stage-game pair $\Gamma[10]$ and $\Gamma[30]$ where the difference $\left|T-T^{\prime}\right|=20$. Recall that for $10 \leq T<20$ the stage game is a Stag hunt $(\mathrm{SH})$ game where the choice $Y$ is weakly (strictly) risk-dominant for $T=10(20)$. For values of $T>20$ the stage game has the structure of a Prisoner's Dilemma (PD) game.

Our treatment variable is the parameterization of $T$, with $T=\{10,30\}$ or $T=\{15,25\}$ and we use a within-subjects design: We vary the order in which the two indefinitely repeated games are played, either the indefinitely repeated Stag Hunt game first followed by the indefinitely repeated Prisoner's Dilemma game, or the reverse order, with the indefinitely repeated Prisoner's Dilemma game first followed by the indefinitely repeated Stag Hunt game. In practice, each session begins with several supergames of the PD version or the $\mathrm{SH}$ version of the stage game. Then we switch the value of $T$ twice (at the start of new supergames only). That is, subjects who started out playing supergames involving the PD game next played several supergames involving the Stag Hunt game and completed the session by playing several supergames involving the original PD game. Subjects who started out playing supergames involving the SH game next played several supergames involving the PD game and finished the session by playing several supergames involving the original SH game. Treatments are denoted by the order of play of the two games and the difference in $T$ between the two games. For example, in Treatment PDSH 20 we start with the PD T $=30$ game and then switch to the $\mathrm{SH} T=10$ game before switching back once more to the PD $T=30$ game. Similarly, in Treatment $S H P D 10$ we start with the SH $T=15$ game and then switch to the PD $T=25$ game and finally switch back to the SH $T=15$ game. 
Table 2: Overview of sessions.

\begin{tabular}{|c|c|c|c|c|c|c|c|}
\hline \multirow[t]{2}{*}{ Treatment } & \multirow{2}{*}{$\begin{array}{c}\text { Session } \\
\text { (chronological order) }\end{array}$} & \multirow[t]{2}{*}{ \#Subjects } & \multirow[t]{2}{*}{ \#Groups } & & \multicolumn{3}{|c|}{ \#Sequences (\#Rounds) } \\
\hline & & & & & Stage 1 & Stage 2 & Stage 3 \\
\hline PDSH 20 & 1 & 10 & 1 & & $6(27)$ & $8(31)$ & $6(33)$ \\
\hline SHPD 20 & 2 & 10 & 1 & & $6(30)$ & $5(32)$ & $9(28)$ \\
\hline PDSH 10 & 3 & 10 & 1 & & $5(30)$ & $6(28)$ & $8(31)$ \\
\hline PDSH 10 & 4 & 20 & 2 & & $4(35)$ & $7(42)$ & $2(10)$ \\
\hline SHPD 10 & 5 & 20 & 2 & & $3(28)$ & $4(29)$ & $6(33)$ \\
\hline PDSH 20 & 6 & 20 & 2 & & $3(37)$ & $7(30)$ & $6(26)$ \\
\hline SHPD 20 & 7 & 20 & 2 & & $3(33)$ & $6(46)$ & $5(21)$ \\
\hline PDSH 10 & 8 & 10 & 1 & & $4(47)$ & $9(30)$ & $3(20)$ \\
\hline SHPD 10 & 9 & 20 & 2 & & $8(19)$ & $4(24)$ & $7(21)$ \\
\hline & Total & 140 & 14 & Avg. & $4.7(31.8)$ & $6.2(32.4)$ & $5.8(24.8)$ \\
\hline
\end{tabular}

In each session, subjects played several supergames (indefinite sequences) of the stage game $\Gamma[T]$ shown in Table 1 . The infinite horizon supergame was constructed as follows. Following play of the stage game subjects took turns rolling a six-sided die. If the die roll came up 1,2, 3, 4 or 5 the stage game was repeated. If the die roll was a six, the supergame was ended. Therefore, the probability of continuation, $p$, was $5 / 6$ and the expected number of rounds in the supergame from any round reached is $1 /(1-p)$ or $6 .^{3}$ This random termination procedure is equivalent to an infinite horizon where the discount factor attached to future payoffs is 5/6 per round (see, e.g., Roth and Murnighan (1978) who originated this methodology). Once a supergame ended, another supergame would begin with the same matching protocol and same population of 10 players used in all previous supergames of the experimental session. Subjects were also informed that at the beginning of each new supergame, the value of $T$ could change, but that the value of $T$ would remain unchanged for all rounds of a supergame.

Table 2 provides an overview of our sessions. Sessions consisted of 10 or 20 subjects. More precisely, at the start of each session, subjects were randomly assigned to a fixed matching group of size 10. Subjects remained in the same matching group with the same other 9 players in all rounds of all supergames played in the session and this fact was public knowledge. In each round subjects were randomly and anonymously paired within their matching group. Subjects were informed of the two possible payoff matrices (either $T=\{10,30\}$ or $T=\{15,25\}$ ) and were instructed that the payoff matrix would not change over the course of each indefinitely repeated sequence of rounds (supergame). However, subjects were instructed that at the start of each new sequence (supergame) the payoff matrix could change. Indeed, to make such a change even more apparent, the payoff matrix for the Stag Hunt game was shown in a red color and the payoff matrix for the Prisoner's Dilemma game was shown in a black color on subjects computer screens. ${ }^{4}$ The duration

\footnotetext{
${ }^{3}$ Allowing subjects to roll a die provides the most credible means of establishing the indefiniteness of the repeated game.

${ }^{4}$ Thus in the very first round of each new supergame it was very evident to subjects whether or not the
} 
of each supergame was common across matching groups in each session but differed across sessions.

Our aim in each session was to conduct approximately 90 total rounds of play and to divide these rounds up so that approximately $1 / 3$ of the rounds involved play of the first game, the next $1 / 3$ involved play of the second game and the final $1 / 3$ involved play of the first game once again. Thus we aimed to get approximately 30 rounds of play with a given payoff matrix before we changed to the other payoff matrix (first stage). Given that the average length of each supergame is 6 rounds, our goal was satisfied by playing, on average, 5 sequences with one game before we changed the game matrix. We repeated the goal of roughly 30 rounds for the second game (second stage) and also for the third and final stage, where the same game was played as in the first stage. As noted above, subjects were completely unaware of our objective of two game changes or of the duration of each of the three stages. They were only instructed that a change could occur at the start of each new supergame (after a die roll of a 6 ). Table 2 indicates some variation in the number of sequences and rounds, which is, of course, due to the randomness of the length of the supergames drawn according to the die rolls.

\subsection{Procedures}

The subjects were recruited from the undergraduate population at the University of Pittsburgh for three-hour sessions. ${ }^{5}$ Subjects had no prior experience participating in any treatment of our experiment. Upon arrival in the laboratory they were randomly assigned to separate computer terminals and received written instructions which were also read aloud in an effort to make the instructions public knowledge. ${ }^{6}$ After instruction, subjects were required to complete a quiz that checked their comprehension of the instructions. A sample copy of the instructions and the quiz is given in Appendix B. After review of the quiz questions and correction of any incorrect quiz answers, subjects began playing an indefinitely repeated version of the stage game shown in Table 1 with a known, treatment specific value of $T$, entering their choices, $X$ or $Y$, on a computer screen when prompted.

Prior to the first round of each new supergame we elicited subjects' beliefs as to the number of the other 9 players in their matching group of size 10 who would choose $X$ in that first round of that supergame. Subjects were aware of the payoff matrix that would be in effect i.e., the value of $T$ each time that we elicited these beliefs. We only elicited beliefs prior to the first round of each new supergame; beyond the first round we did not elicit beliefs so as to avoid being too intrusive regarding subjects' decision processes and delaying completion of the experiment in a timely manner. Moreover, we did not incentivize this belief elicitation to avoid further complicating the game; nevertheless, as we shall discuss below, the beliefs we elicited are very informative about subsequent play. Beliefs were elicited in all sessions

payoff parameter, $T$, of the stage game had changed.

${ }^{5}$ We typically finished a session well before this limit (in about 1.5 hours or less) so as to avoid possible end game effects associated with the finite 3-hour time horizon of a session.

${ }^{6}$ The experiment was conducted using z-tree (Fischbacher, 2007). 
except the first two sessions we ran (sessions 1 and 2 in Table 2).

Following the input of their belief in the first round of each new supergame, subjects played the round by choosing $X$ or $Y$. At the end of the first round and each subsequent round of the supergame, subjects were reminded of the game matrix and of their own choice for the round and were also informed about their matched player's choice, and their own and their matched player's payoffs for the round. Then a six-sided die was rolled to determine whether the supergame would continue with another round. Subjects took turns rolling the die. Subjects were provided with a complete history of their own past play from all rounds of all prior supergames. The game payoffs in Table 1 represent the monetary payoffs in terms of cents (US\$). Subjects were paid their payoffs from all rounds of all supergames they played and in addition they were given a show-up payment of $\$ 5$. Total earnings for subjects averaged about $\$ 17$ (including the $\$ 5$ show-up fee), and sessions typically lasted about 90 minutes.

\subsection{Hypotheses}

Our experiment was designed to test two main hypotheses. The first hypothesis concerns the role of precedents.

Hypothesis 1 A precedent for equilibrium selection in game $\Gamma[T]$ carries over to a similar game, $\Gamma\left[T^{\prime}\right]$, where $T^{\prime} \neq T$ and all other elements of the two games are held constant.

By equilibria, we have in mind the two, population-wide pure-strategy equilibria, where all play $X$ or all play $Y$, though we recognize that there exist other equilibria in the environments that we study. If play settles on one of these two pure equilibria in the first of the two games played, then we hypothesize that this precedent serves as a criterion for selecting the same pure equilibrium in the second, similar game despite the change in the temptation value, T. Our second hypothesis pertains to variations in the similarity between the two games as measured by the difference in the temptation payoff values.

Hypothesis 2 The more similar are the two games (the smaller is $\left|T-T^{\prime}\right|$ ), the greater is the role played by precedents for equilibrium selection between the two games.

The more similar are the two stage games, the more likely it is that a precedent for equilibrium selection in one game carries over to the other similar game. The logic here follows from a simple continuity argument; if the difference between the two temptation payoffs were 0 , the games would not differ at all and thus once an equilibrium was selected the population of players would likely remain at that same equilibrium forever after. However as the difference in the temptation values grows, the strategic incentives of the two games begin to differ and precedents for equilibrium selection in one game may become weaker for equilibrium selection in the other, similar game. Thus we predict that precedents may play a greater role in our $T=\{15,25\}$ treatment where the two stage games are more similar in payoff incentives as compared with our $T=\{10,30\}$ treatment where the two stage games are more different in payoff incentives. 


\section{Results}

\subsection{Cooperation in less similar games - PDSH 20 and $S H P D 20$}

We start our analysis with a detailed look at first-round choices and beliefs of subjects in games where $\left|T-T^{\prime}\right|$ is large, i.e., $T=\{10,30\}$. Table 3 displays the average cooperation rate (average choice of $X$ ) and beliefs about others' play of $X$ in the first round of a sequence separately for each stage. The columns in Table 3 labeled "Choice $X$, round 1" report the aggregate frequency of cooperative play in the first round of the first sequence in a stage for each group. Recall that each stage consists of several sequences of rounds (supergames) of the same game and columns labeled "Choice $X$, all first rounds" show the frequency of cooperative play in first rounds of all sequences in a stage. Similarly, the last six columns report subjects' beliefs about the frequency of cooperation in their matching group in the first round of the first sequence in a stage (columns 7-9) and first rounds of all sequences for each stage (columns 10-12).

As Table 3 reveals, there are clear differences in behavior between PDSH 20 and SHPD 20 . Beginning with the first round of the first supergame in stage 1, we observe that cooperative play (i.e., the choice of $X$ ) is higher in $S H P D 20$ where subjects start with the stag-hunt game (SH) than in PDSH 20 where subjects start with the Prisoner's Dilemma game (PD) (0.87 vs. 0.60). According to Fisher's exact test, the hypothesis that cooperative play is equally likely in both the PD and SH can be rejected $(p=0.039)$. This is also reflected in subjects' beliefs (Table 3, column 7). In PDSH 20 subjects expect less cooperative choices $(X)$ from other subjects in their matching group than in SHPD $20(p<0.01$, two-sided t-test).

When the game changes in the first round of stage 2 from the PD to the SH (in $P D S H$ 20), the first-round cooperation rate in the second stage is higher than in the first round of stage 1. This difference is also reflected in subjects' beliefs, which reveal that they expect most others to cooperate in the SH (Table 3, column 8), but not so much in the PD (Table 3, column 7). A reverse picture emerges in SHPD 20. Switching from SH to a PD results in lower cooperation rates in the first round of stage 2 than in the first round of stage 1. Again, first-round choices in SHPD 20 are reflected in subjects' beliefs (Table 3, column 8). Interestingly, it seems that previous experience plays only a minor role as evidenced by a comparison of stage $1, \mathrm{PD}$ and stage $2, \mathrm{PD}$ and stage $1, \mathrm{SH}$ and stage 2, $\mathrm{SH}$. We cannot reject the hypothesis of an equal distribution of choices in the first round of the stage $1, \mathrm{PD}$ and stage 2, PD (Fisher's exact test, $p=0.196$ ) and the same finding holds for stage 1, SH and stage 2, SH (Fisher's exact test, $p=1.000$ ).

Comparing the first round in a stage with all first rounds of all sequences in a stage gives a first glimpse as to how behavior unfolds over time. In general, we observe low cooperation rates over all first rounds accompanied by low expectations about other players' cooperation in the PD's indicating that at the start of a new sequence cooperation rates and beliefs decline over time, whereas in the $\mathrm{SH}$ we observe the opposite pattern. The first-round cooperation rates and beliefs in the SH's typically do not decline over a stage (see columns labeled "Choice $X$, all first rounds", Table 3). Indeed, these cooperation rates are in most 


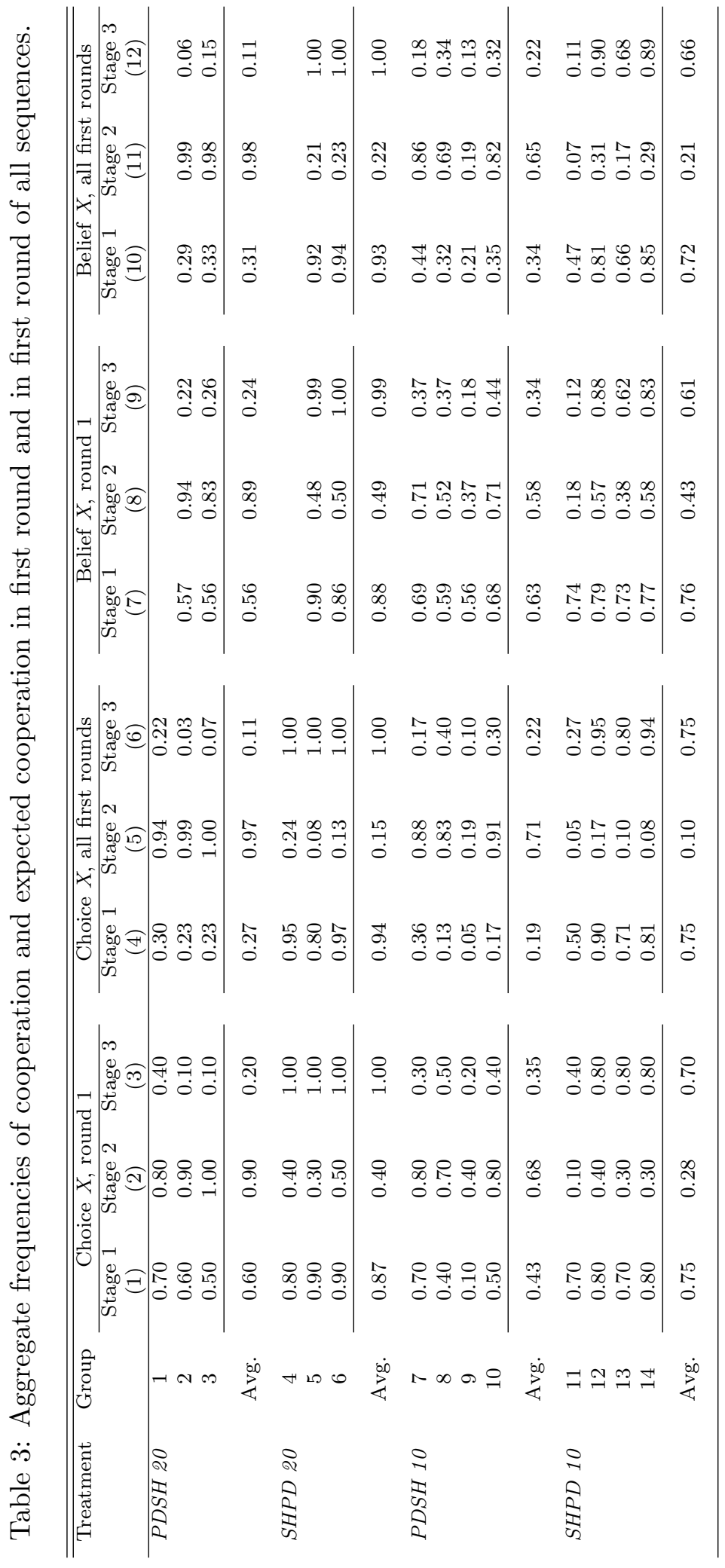


cases higher than in the first round of a stage. In summary, the first-round behavior indicates two very distinct behavioral patterns in the $\mathrm{PD}$ and $\mathrm{SH}$ games and so we next turn to choices made in all rounds of all sequences of the three stages.

Table 4 displays the aggregate frequency of cooperative play in each group along with the averages in the respective treatment in the first three columns. In the last three columns we report the aggregate frequency of coordination (i.e., the play of either $X X$ or $Y Y$ by randomly matched pairs of subjects).

The top panel of Figure 1(a) presents data on the aggregate frequency of cooperative play (i.e., the choice of $X$ ) in each round of a stage of the three groups that started with the PD in stage 1 (treatment PDSH 20). The bottom panel of Figure 1(a) presents the aggregate frequency of cooperation in each round of a stage of the three groups that started with the SH in stage 1 (treatment SHPD 20). In Figure 1(b) the left (right) panel shows the frequency of cooperative play for each group separately in PDSH 20 (SHPD 20). Each stage consists of several sequences of rounds (or supergames) and within a stage the game matrix was always the same. That is, in PDSH 20 groups played several sequences of the PD in stage 1 followed by several sequences of the SH in stage 2 and finally several sequences of the PD in stage 3, whereas in SHPD 20 groups played several sequences of the $\mathrm{SH}$ in stage 1 , then several sequences of the PD in stage 2 followed again be several sequences of the SH in stage 3 . In each figure, the $\mathrm{x}$-axis reports the consecutively numbered rounds within a stage so that a round number of 1 indicates the start of a new stage, i.e., a change in the temptation payoff, $T$. Additionally, in Figure 1(b) the start of a new sequence (supergame) is indicated by the gray dashed vertical lines and the start of a new stage is indicated by red dashed vertical lines. Note that the average duration of stages 1 and 2 is about 32 rounds and the duration of stage 3 averages about 25 rounds (see also Table 2). Thus, the aggregate frequencies in the later rounds of a stage in Figure 1(a) do not necessarily include choices from all groups in a treatment, due to different supergame lengths in each stage.

Figure 1(a) shows that the differences in first-round cooperation rates of the first stage extend to future rounds and become even more pronounced. Indeed, while cooperative play increases further in SHPD 20 reaching full cooperation in round 7, the opposite is true in $P D S H$ 20. Within a few rounds the frequency of cooperation plummets toward zero in $P D S H$ 20. These time patterns are reflected in the average frequency of cooperative play over all rounds in stage 1 as reported in Table 4. All three groups in SHPD 20 show substantially higher cooperation rates than the three groups in $P D S H 20$ and thus, subjects have markedly different experiences in the two treatments (see Figure 1(b)). They experienced high rates of defection (i.e., choice of $Y$ ) in the stage 1, PD in PDSH 20 and high rates of cooperation in the stage 1, SH in SHPD 20.

There is a dramatic change in behavior once the temptation payoff $T$ is changed. The change of $T$ occurs in both treatments after about 30 rounds of play. In PDSH 20, we observe a sudden increase of cooperative play from below 0.1 in the last round of the last sequence in stage 1 to 0.9 in the first round of the first sequence in stage 2. Surprisingly, the same subjects who were not able to sustain cooperative play in the PD are rather confident, as indicated by their beliefs, that their opponents will choose the Pareto-efficient action 

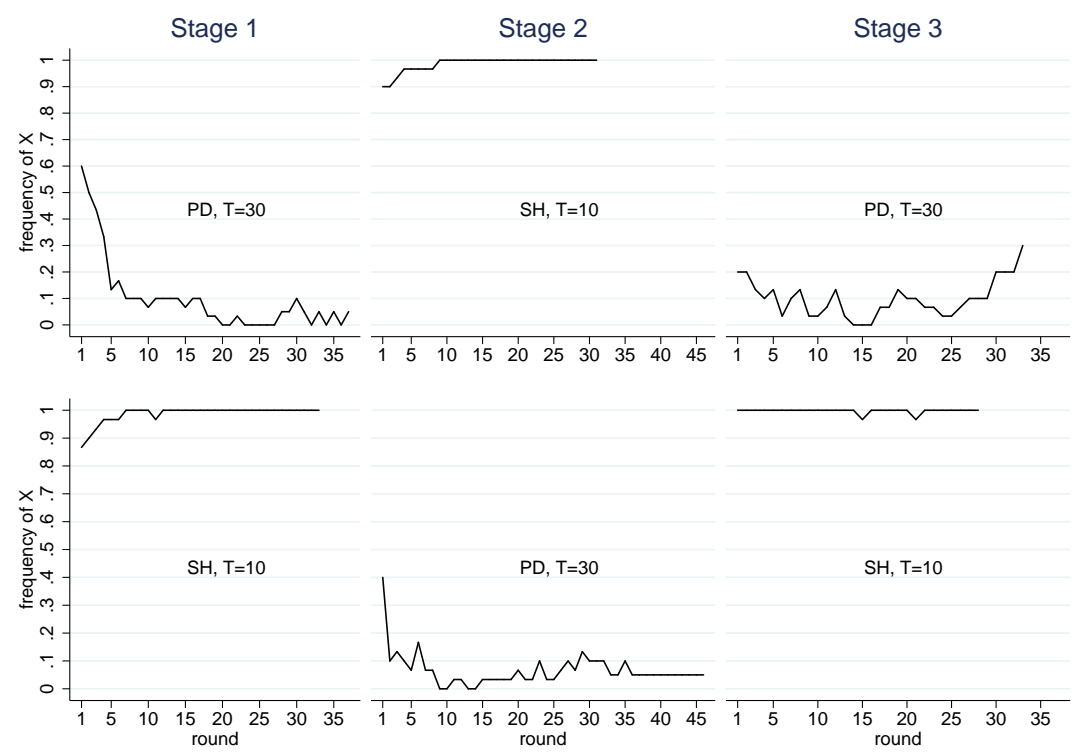

(a) Aggregate frequency of cooperation in PDSH 20 (top panel) and SHPD 20 (bottom panel).
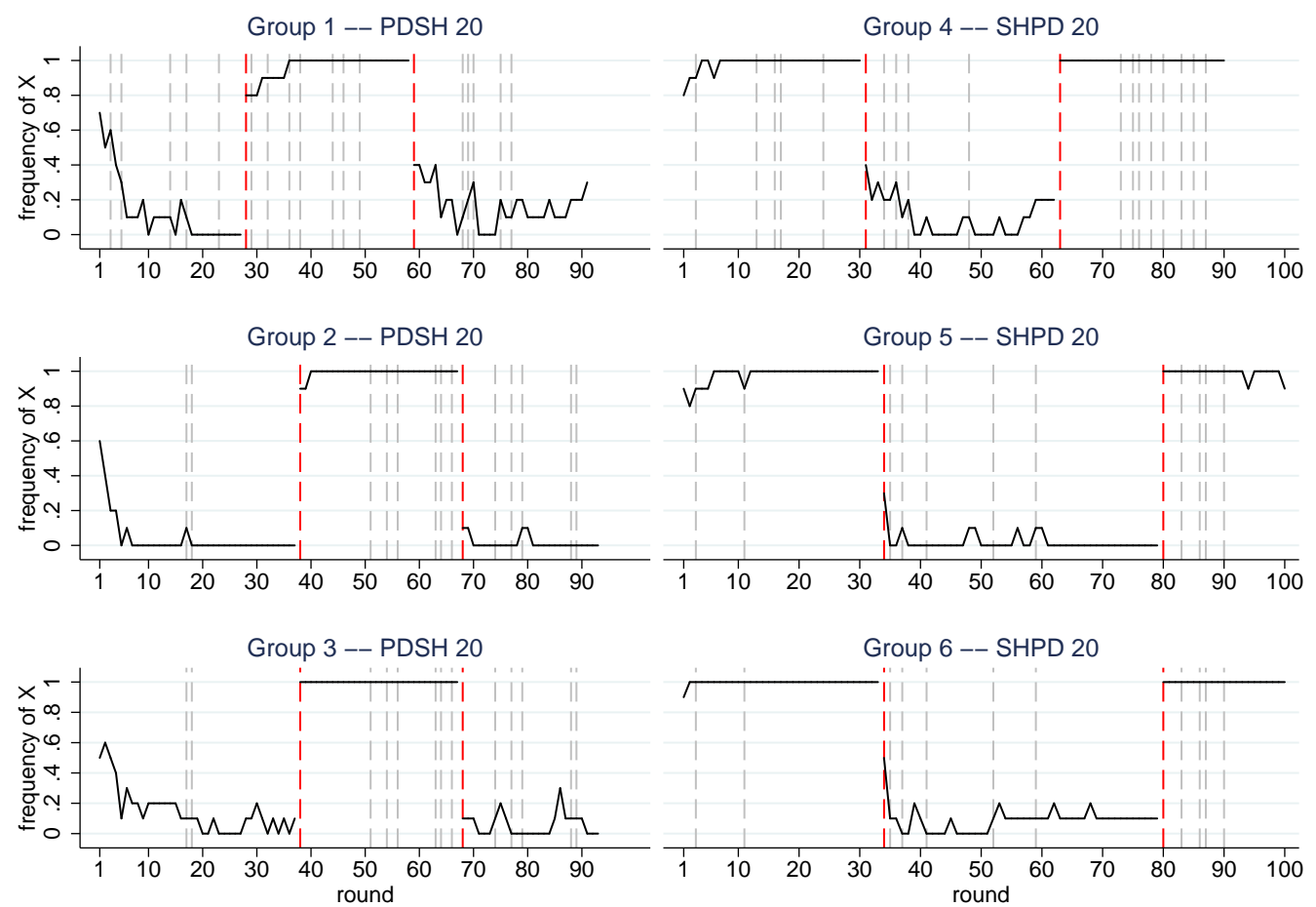

(b) Aggregate frequency of cooperation at the group level - PDSH 20 (left panel) and SHPD 20 (right panel).

Figure 1: Frequency of cooperation in PDSH 20 and SHPD 20 
Table 4: Aggregate results: Frequencies of cooperation and coordination in PDSH 20 and SHPD 20

\begin{tabular}{|c|c|c|c|c|c|c|c|}
\hline \multirow{2}{*}{ Treatment } & \multirow[t]{2}{*}{ Group } & \multicolumn{3}{|c|}{ Choice $X$} & \multicolumn{3}{|c|}{ Coordination } \\
\hline & & Stage 1 & Stage 2 & Stage 3 & Stage 1 & Stage 2 & Stage 3 \\
\hline \multirow[t]{4}{*}{ PDSH 20} & 1 & 0.14 & 0.96 & 0.17 & 0.83 & 0.94 & 0.74 \\
\hline & 2 & 0.04 & 0.99 & 0.02 & 0.92 & 0.99 & 0.97 \\
\hline & 3 & 0.15 & 1.00 & 0.06 & 0.79 & 1.00 & 0.88 \\
\hline & Avg. & 0.11 & 0.99 & 0.09 & 0.85 & 0.98 & 0.85 \\
\hline \multirow[t]{4}{*}{ SHPD 20} & 4 & 0.98 & 0.10 & 1.00 & 0.97 & 0.81 & 1.00 \\
\hline & 5 & 0.98 & 0.02 & 0.99 & 0.96 & 0.96 & 0.98 \\
\hline & 6 & 1.00 & 0.09 & 1.00 & 0.99 & 0.83 & 1.00 \\
\hline & Avg. & 0.99 & 0.07 & 1.00 & 0.97 & 0.87 & 0.99 \\
\hline \multirow[t]{5}{*}{ PDSH 10} & 7 & 0.30 & 0.89 & 0.13 & 0.59 & 0.79 & 0.77 \\
\hline & 8 & 0.05 & 0.78 & 0.18 & 0.90 & 0.62 & 0.72 \\
\hline & 9 & 0.04 & 0.09 & 0.03 & 0.93 & 0.84 & 0.94 \\
\hline & 10 & 0.06 & 0.91 & 0.10 & 0.89 & 0.84 & 0.87 \\
\hline & Avg. & 0.10 & 0.63 & 0.12 & 0.84 & 0.77 & 0.81 \\
\hline \multirow[t]{5}{*}{ SHPD 10} & 11 & 0.24 & 0.02 & 0.11 & 0.64 & 0.97 & 0.85 \\
\hline & 12 & 0.97 & 0.08 & 0.99 & 0.94 & 0.86 & 0.98 \\
\hline & 13 & 0.71 & 0.08 & 0.80 & 0.48 & 0.88 & 0.71 \\
\hline & 14 & 0.85 & 0.03 & 0.95 & 0.72 & 0.94 & 0.90 \\
\hline & Avg. & 0.68 & 0.05 & 0.67 & 0.71 & 0.91 & 0.87 \\
\hline
\end{tabular}

once the temptation payoff $T$ is lowered. Over all rounds, we observe the same frequency of cooperative play in stage 2, SH (in PDSH 20) and stage 1, SH (in SHPD 20). Thus, it seems that it does not matter whether subjects are inexperienced as in SHPD 20 or whether subjects experienced high rates of defection, and thus a high level of mistrust, before they play the $\mathrm{SH}$ as in PDSH 20. By contrast, in SHPD 20, where subjects experienced cooperative behavior throughout stage 1 , the frequency of cooperative play immediately dropped from perfect cooperation (1.0) to 0.4 and never rebounds in subsequent rounds. Again, previous experience has no effect on play in the stage 2 game. That is, despite the fact that subjects in SHPD 20 experienced cooperation throughout stage 1, the average cooperation rates in the three groups participating in the stage 2, PD are about as low as in the three groups who participated in the stage 1, PD in PDSH 20.

In stage 3 the frequency of cooperative play reverts, in both treatments, to observed stage 1 levels. In $P D S H$ 20, confidence in cooperative play is immediately lost when the temptation payoff $T$ is increased again to 30, i.e., the frequency of cooperative play in $P D S H$ 20 plummets to 0.2 and fluctuates between 0 and 0.2 in the remaining rounds of stage 3 . On the other hand, confidence is immediately restored in SHPD 20 when $T$ is lowered to 10 and we observe full cooperation in the first round and almost all subsequent rounds in stage 3. 
Clearly, the analysis suggests that there are no order effects, i.e., play is unaffected by previous experience in a similar game. In fact, Table 4 reveals that the aggregate frequency of cooperative play in the PD's and SH's is similar across stages (between 0.07 and 0.11 for the PD's and between 0.99 and 1.0 for the SH's).

Table 4 also reports the coordination rates in PDSH 20 and SHPD 20 in the last three columns. The coordination rate measures the frequency of either $X X$ or $Y Y$ play in a group and thus gives us an indication of the extent of pure equilibrium play. While the coordination rates are well above 0.7 , in all stages of both $P D S H 20$ and SHPD 20, the underlying behavior in the PD and $\mathrm{SH}$ is quite different. In the $\mathrm{SH}$ we observe coordination rates close to 1.0 (between 0.94 to 1 ) indicating almost perfect coordination on $X$. Unlike the $\mathrm{SH}$, the coordination rates in the PD are somewhat lower, between 0.74 and 0.96 , and imply that most, though not all subject pairs coordinate on the non-cooperative choice $Y$. The lower coordination rates in the PD also indicate attempts to induce cooperative play in the group. However, these attempts remain largely unsuccessful since the frequency of $\mathrm{X}$ choices is too low to turn play around. We can reject the hypothesis of no difference in coordination rates between the two treatments in all three stages using robust rank-order tests (all p-values $p \leq 0.05){ }^{7}$

Based on these observations, we can clearly reject Hypothesis 1 for the treatment with $T=\{10,30\}$. That is, we find no evidence that a precedent for equilibrium selection in game $\Gamma[30]$ carries over to a similar game, $\Gamma[10]$ and also not from game $\Gamma[10]$ to game, $\Gamma[30]$. Apparently, once the incentives to deviate from cooperation are sufficiently low as in stage 2 of $P D S H$ 20, there is no scope for a transfer of a precedent of defection. In a similar vein, a precedent of cooperation does not transfer to a similar game in which the incentives to deviate from cooperation are high as in stage 2 of SHPD 20.

\subsection{Cooperation in more similar games - PDSH 10 and $S H P D$ 10}

We have provided evidence that the value of $T$ plays a crucial role for equilibrium selection and effectively gives no room for a transfer of precedents. In treatments PDSH 10 and SHPD 10 the difference $\left|T-T^{\prime}\right|$ is smaller than in treatments PDSH 20 and SHPD 20 and thus the two stage games $\Gamma[25]$ and $\Gamma[15]$ are more similar.

We again start with behavior in the first round of the first sequence of stage 1 . Despite the greater similarity between the stage games, we observe that cooperation rates in the SH are at least as high and in most cases higher than in the PD. When subjects start with the PD (in PDSH 10) about 43 percent choose $X$ in the first round of the first sequence compared with about 75 percent when subjects start with the SH (in SHPD 10). Using Fisher's exact test, we can reject the hypothesis of an equal distribution of cooperative play $(\mathrm{p}=0.006)$. On average, the observed choices correspond to subjects' stated beliefs. They expect significantly more cooperative play in the first round of the $\mathrm{SH}$ than in the first round

\footnotetext{
${ }^{7}$ See Feltovich (2003) for a discussion of the robust rank-order test.
} 
of the $\mathrm{PD}(p<0.01$, two-sided t-test $)$.

Notice that the initial cooperation rate is not only lower in the $\mathrm{PD}, T=25$ (0.43) than in the $\mathrm{PD}, T=30$ (0.60) but also in the $\mathrm{SH}, T=15(0.75)$ than in the $\mathrm{SH}, T=10$ (0.87). However, neither difference is statistically significant according to Fisher's exact tests $(p>0.22)$. Thus, small differences in $T$, that is either a lower incentive to deviate from cooperation in the $\mathrm{PD}$ or a higher risk of choosing $X$ in the $\mathrm{SH}$, are not initially taken into account by subjects. This is corroborated by subjects' beliefs. On average, subjects expect cooperative play to be approximately the same in the $\mathrm{PD}, T=30(0.56)$ as in the $\mathrm{PD}, T=25$ (0.63) $(p>0.31$, two-sided t-test). In the $\mathrm{SH}$ games, subjects' expectations are on average closer to actual observed behavior. Indeed, they expect a bit more cooperation in the SH, $T=10$ (0.88) than in SH, $T=15$ (0.76). The difference is statistically significant at the margin $(p=0.094$, two-sided t-test).

In stage 2, first-round cooperation is higher (lower) than in the first round of the first sequence in PDSH 10 (SHPD 10) in all four groups (see Table 3). At a first glance, previous experience seem to matter for initial choices in stage 2 . In particular, for the $\mathrm{PD}, T=25$ we observe that inexperienced subjects $(P D S H 10)$ choose $X$ to a greater extent than subjects who were previously exposed to an environment with high cooperation (in $S H P D$ 10)(0.43 vs. 0.28). Likewise, we observe that in $\mathrm{SH}, T=15$ inexperienced subjects ( $S H P D$ 10) choose $X$ to a somewhat greater extent than subjects who were previously exposed to an environment with low cooperation (in $P D S H$ 10) (0.75 vs. 0.68). However, we cannot reject the hypothesis of an equal distribution of choices in the first round of the stage 1, PD and stage 2, PD (Fisher's exact test, $p=0.241$ ) and also not for the stage 1 , SH and stage 2, SH (Fisher's exact test, $p=0.622$ ).

Extending our view to all first rounds reveals that cooperative play over all first rounds of a PD is lower than in the initial round of a stage in all groups and all stages (Table 3, column 4-6). The behavior in the $\mathrm{SH}$ is less clear cut. It appears that in two groups (groups 9 and 11) cooperation rates over all first rounds in the $\mathrm{SH}$ are lower indicating that choices of $X$ decline over time. In the remaining six groups, cooperation rates over all first rounds are as least as high or higher than in the first round of a stage.

We now turn to a more detailed look on how behavior unfolds over time. The top panel of Figure 2(a) presents data on the aggregate frequency of cooperative play in each round of a stage of the four groups that started with the PD in stage 1 in PDSH 10. The bottom panel of Figure 2(a) presents the aggregate frequency of cooperation in each round of a stage of the four groups that started with the SH in stage 1 in SHPD 10. Again, Figure 2(b) shows the frequency of cooperative play for each group in $P D S H 10$ in the left panel and for each group in $S H P D 10$ in right panel. Figure 2 is constructed in the same way as Figure 1 . The $\mathrm{x}$-axis reports the consecutively numbered rounds within a stage. Thus a round number of 1 indicates the start of a new stage, i.e., a change of the temptation payoff $T$, but within each stage many indefinitely repeated supergames may have been played (see Table 2 for the precise numbers). In Figure 2(b) a start of a new sequence is indicated by the gray dashed vertical lines and a start of a new stage is indicated by the red dashed vertical lines.

Figure 2(a) shows that as subjects gain experience in the stage 1, PD, cooperation rates 

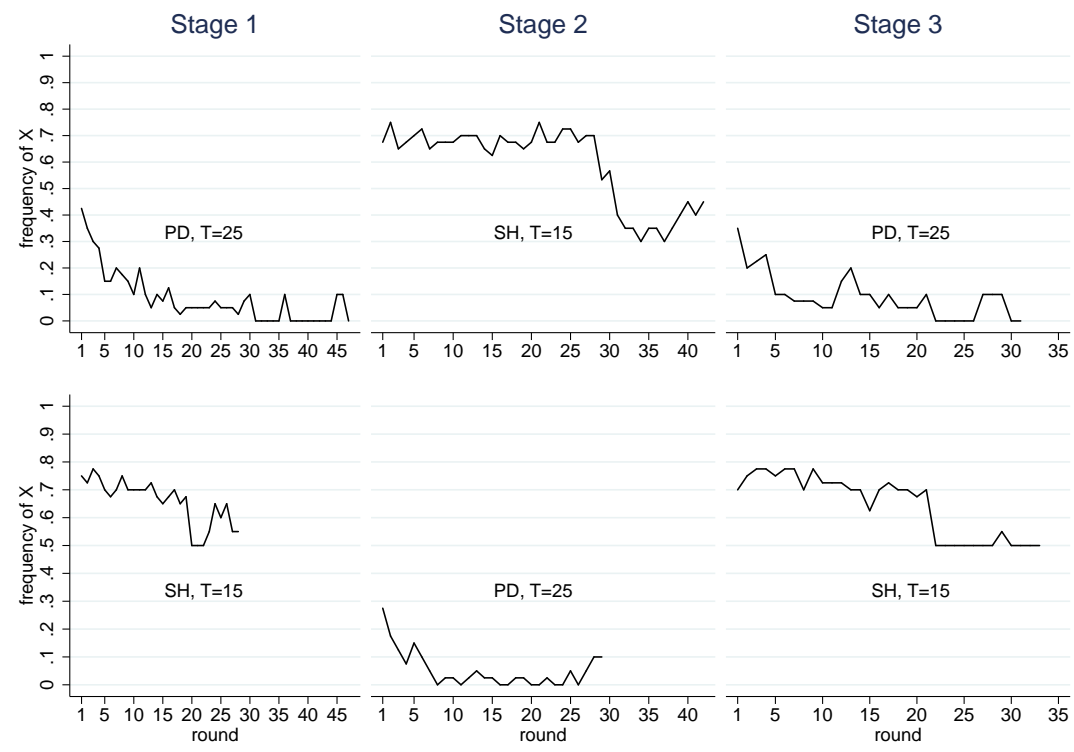

(a) Aggregate frequency of cooperation in $P D S H 10$ (top panel) and SHPD 10 (bottom panel).
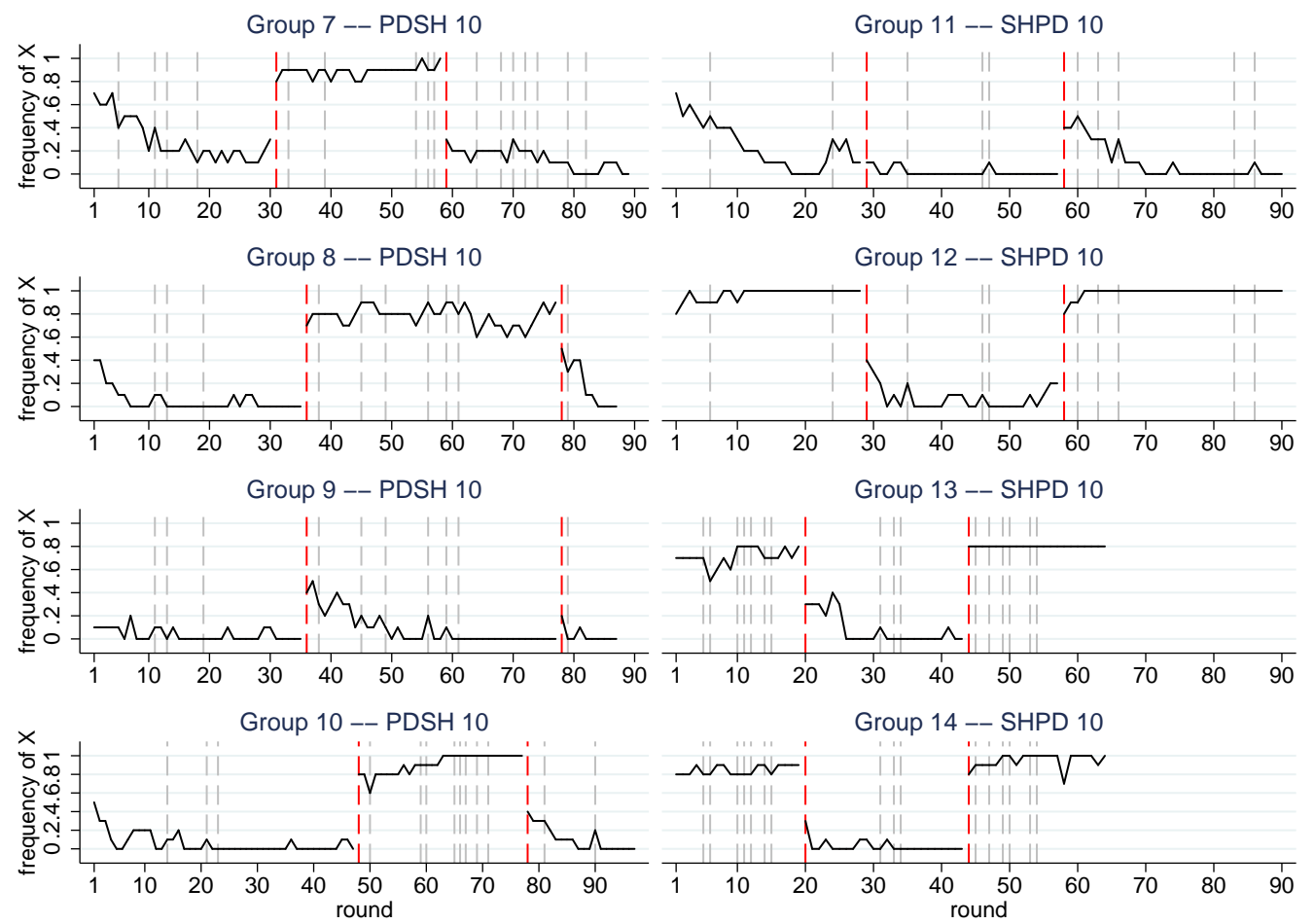

(b) Aggregate frequency of cooperation at the group level - PDSH 10 (left panel) and SHPD 10 (right panel).

Figure 2: Frequency of cooperation in PDSH 10 and SHPD 10 
decay and converge toward zero in the later rounds of that first stage. This is similar to the stage 1, PD in PDSH 20 where cooperation quickly converges to zero as well. Such similarities are, however, not the case in the SH. In contrast to stage $1 \mathrm{SH}$ play in $S H P D$ 20, where play attained full cooperation over time, we observe a slow decay of cooperation rates in the stage $1 \mathrm{SH}$ of $S H P D$ 10, where the cooperation rates approach 0.5 toward the later rounds of stage 1 .

A change in $T$ results in sudden swings of behavior as previously seen in PDSH 20 and $S H P D$ 20. Almost 70 percent of subjects switch from uncooperative to cooperative play in the first round of stage 2 when $T$ is lowered from 25 to 15 in PDSH 10. The cooperation rate remains stable throughout most of the rounds of stage 2. A reverse picture obtains in SHPD 10 where $T$ is increased from 15 to 25 at the beginning of stage 2 . Cooperative play immediately collapses and reaches a frequency of cooperation of 0.1 or below within a few rounds. In stage 3 we observe in both PDSH 10 and SHPD 10 the same patterns as in stage 1. Table 4 reveals that once again there is no evidence for order effects; the aggregate frequency of cooperative play in PD's are comparable across stage $1-3(0.10,0.05$ and 0.12$)$ as well as for the SH's (0.68, 0.63 and 0.67).

The coordination rates displayed in the last three columns of Table 4 reveal that on average subjects are less coordinated in the $\mathrm{SH}$ than in the PD. In the PD's coordination rates are mostly above 0.86 , whereas in the SH's they are mostly below 0.86. In the PD, subjects coordinate in most cases quickly on mutual defection, $Y Y$. In $\mathrm{SH}$, however, it seems that the risk dominant choice, $Y$, creates some tension between choosing $X$ and $Y$ resulting in more frequent miscoordination.

The evidence thus far suggests that while observed behavior in the PD is quite consistent in both PDSH 10 and SHPD 10, there are some differences for the SH. This is confirmed by looking more closely at the group level, cooperation rates. Figure 2(b) reveals that a majority of groups display a high cooperation rate in the $\mathrm{SH}$. In fact, this is the case when the $\mathrm{SH}$, $T=15$ is played first and even after experiencing low cooperation rates in the $\mathrm{PD}, T=25$. However, in two groups (group 9 in PDSH 10 and group 11 in SHPD 10) cooperation rates in the $\mathrm{SH}$ are low from the very beginning and decline towards zero cooperation over time. In group 9 involving treatment $P D S H 10$ it appears that a precedent of defection in the first $\mathrm{PD}$ supergames transfers to the SH supergames in stage 2. While the transfer of play of $Y$ holds for a majority of subjects in this group, there were also 4 subjects who switched to cooperative play of $X$ when the change to $\mathrm{SH}$ occurred. These are, of course, too few subjects to push play towards the cooperative equilibrium and thus we see a convergence to all $Y$ within 15 rounds. In stage 3 (PD) almost all (8 out of 10) continue to play their previous stage 2 , action $(Y)$. In the other group - group 11 involving treatment SHPD 10 - we again observe evidence for a precedent transfer. This group started out with the SH, $T=15$ in stage 1, in which play converged towards the inefficient, all $Y$ equilibrium. When the game was changed to the PD in stage 2, subjects continue to play $Y$ and the frequency of cooperative play is virtually zero throughout this second stage. Notice, that in contrast to all other repeated PD's in a similar situation, the cooperation rate in the first round of stage 2 is very low, indicating no attempt to cooperate. In stage 3, a minority of subjects 
switch from playing $Y$ to $X$ in the beginning, which, however, does not prevent the group from converging back to nearly unanimous play of $Y$ again.

To summarize the above discussion, we do not find strong evidence that equilibrium selection in one repeated game serves as a precedent for equilibrium selection in a similar repeated game, despite the fact that all playing $X$ or all playing $Y$ are equilibrium possibilities in both indefinitely repeated games. Thus, there is not much in the way of support for our Hypothesis 1 for these games either.

\subsection{Comparing behavior across treatments}

Our second hypothesis was that more similar games, i.e., games with a smaller difference $\left|T-T^{\prime}\right|$, facilitate the transfer of a precedent. To investigate this hypothesis we compare behavior across treatments, i.e., PDSH 20 and SHPD 20 versus PDSH 10 and SHPD 10. Let us first concentrate on how different values of $T$ affect behavior in either the $\mathrm{PD}$ or the SH. The previous analysis in PDSH 20 has shown that there is some initial cooperation in the PD game followed by a strong increase in defection. Surprisingly, a lower temptation payoff $(T=25)$ does not lead to less defection. Even though the gain from deviating from the cooperative choice when $T=25$ is smaller as compared to when $T=30$, cooperation is nor more easily sustained in the PD game with a lower $T$. Using a robust rank-order test we cannot reject the hypothesis of no difference in cooperation rates in stages 1, 2 and 3 PD's between PDSH 20 and PDSH 10 (all p-values $p=0.114$ ).

To the contrary, a higher temptation payoff $(T=15)$ in the SH does have an effect on behavior. A higher $T$ makes play of $X$ less attractive (risk-dominated) and subjects appear to react to this circumstance with a lower cooperation rate when $T=15$. The hypothesis of no difference in cooperation rates in stage 1, 2 and 3 SH's between PDSH 20 and PDSH 10 can be clearly rejected according to a robust rank-order test (for all three stages $p \leq 0.029$ ). ${ }^{8}$

These findings suggest that in contrast to Hypothesis 2, it does not appear that precedent plays a stronger role when the difference in the temptation payoffs of the two games is smaller, so that the two games are more similar. While cooperation in the $\mathrm{SH}, T=15$ is indeed lower following a precedent of inefficient play in the PD, $T=25$ as compared to cooperation in the $\mathrm{SH}, T=10$ following a precedent of inefficient play in the $\mathrm{PD}, T=30$, it is also the case that cooperation is lower when the $\mathrm{SH}, T=15$ is the first game played as compared with the case where $\mathrm{SH}, T=10$ is the first game played - see, e.g., Figures 1 and 2. Thus it appears that risk dominance considerations rather than equilibrium selection precedents or similarity measures plays the more important role in understanding the choices made by subjects in our experiment.

On the other hand, in support of Hypothesis 2, we note that the only two instances in which a transfer of precedent for equilibrium selection took place were in treatments where the two games were more similar to one another. In particular, we observe one instance

\footnotetext{
${ }^{8}$ It is important to note that this result does not depend on the inclusion of the two groups with a low cooperation rate (group 9 and 11). As Table 4 shows, cooperation rates in $\mathrm{SH}, T=15$ are in all stages lower than in $\mathrm{SH}, T=10$ for each group.
} 
(group 11) in SHPD 10 where a precedent of inefficient play in the SH transfered to the $\mathrm{PD}$, and one instance (group 9) in $P D S H$ 10, where a precedent for inefficient play in the $\mathrm{PD}$ transfered to the $\mathrm{SH}$. There are no instances where a precedent for equilibrium selection transfers between different games played in the SHPD 20 or PDSH 20 treatments. Finally, we note that we do not observe any cases where a precedent for efficient play (all $X$ ) in the $\mathrm{SH}$ transfers over to a subsequent PD; it seems that only precedents for inefficient equilibrium play (all $Y$ ) have some spillover effects in more similar repeated games.

\section{Individual behavior across treatments}

This section takes a closer look at individual behavior in order to shed light on why precedents are often ineffective and do not generally transfer from game to game, even when the difference in $T$ is small so that the games are more similar. We first provide a more thorough analysis of elicited beliefs. That is, we look at best-response behavior and how well beliefs are calibrated to actual behavior in the first round of a particular sequence. We then examine cooperation frequencies at the individual level and analyze the factors that may have induced subjects to change their action choices. Finally, we study choices in the first round of a new stage, i.e., stage 2 or 3 . Utilizing the belief data we provide insights that help explain the sudden swings in behavior that accompany changes in $T$.

\subsection{Best response behavior and accuracy of beliefs}

We elicited subjects' beliefs about cooperative play in a matching group in the first round of a sequence except in the first session of PDSH 20 and SHPD 20. Up to now, we have only provided some coarse assessment of how informative elicited beliefs are for observed play. Table 5 gives a more detailed overview and shows best-response rates and the accuracy of beliefs for each stage and for both treatments and both orders.

Notice first that following the logic of Kandori (1992) (see Appendix A), cooperation in the infinitely repeated game can be a best response if a player believes that all other players in his group will cooperate. ${ }^{9}$ It follows that a subject is off-the-equilibrium path, i.e. plays a non-best response in the PD if he plays $X$ but does not expect cooperation from all other players. By contrast, in a $\mathrm{SH}$, a non-best response can involve play of both $X$ and $Y$, depending on beliefs. Therefore, given the previous evidence we have seen for PDSH 20 and $S H P D$ 20, it is not surprising that best-response rates are lower in PD's than in SH's. In the $\mathrm{SH}$ we observe close to perfect best-response behavior in all stages, reflecting the fact that groups either start out in the cooperative equilibrium (stage 1) or quickly transition to play of all $X$ in stage 2 and 3. In the PD's, however, best response rates are considerably lower. In particular, in the stage $1, \mathrm{PD}$ game they are below 80 percent indicating a significant

\footnotetext{
${ }^{9}$ The idea behind Kandori's contagious equilibrium is that cooperation can be sustained only if all players cooperate because defection by a single player would initiate a contagious spread of defection in the entire community (group) and this process cannot be stopped by re-igniting cooperation.
} 
Table 5: Aggregate best response behavior and accuracy of beliefs

\begin{tabular}{|c|c|c|c|c|c|c|c|}
\hline \multirow[t]{2}{*}{ Treatment } & \multirow[t]{2}{*}{ Group } & \multicolumn{3}{|c|}{ best response } & \multicolumn{3}{|c|}{ accuracy } \\
\hline & & Stage 1 & Stage 2 & Stage 3 & Stage 1 & Stage 2 & Stage 3 \\
\hline \multirow[t]{4}{*}{ PDSH 20} & 1 & & & & & & \\
\hline & 2 & 0.77 & 1.00 & 0.98 & 0.22 & 0.02 & 0.08 \\
\hline & 3 & 0.77 & 0.99 & 0.93 & 0.16 & 0.01 & 0.13 \\
\hline & Avg. & 0.77 & 0.99 & 0.96 & 0.19 & 0.02 & 0.10 \\
\hline \multirow[t]{4}{*}{ SHPD 20} & 4 & & & & & & \\
\hline & 5 & 0.97 & 0.92 & 1.00 & 0.14 & 0.17 & 0.00 \\
\hline & 6 & 0.97 & 0.88 & 1.00 & 0.02 & 0.18 & 0.00 \\
\hline & Avg. & 0.97 & 0.90 & 1.00 & 0.08 & 0.17 & 0.00 \\
\hline \multirow[t]{5}{*}{ PDSH 10} & 7 & 0.68 & 0.85 & 0.82 & 0.22 & 0.18 & 0.18 \\
\hline & 8 & 0.90 & 0.66 & 0.60 & 0.18 & 0.22 & 0.30 \\
\hline & 9 & 0.95 & 0.83 & 0.90 & 0.14 & 0.25 & 0.15 \\
\hline & 10 & 0.82 & 0.81 & 0.70 & 0.21 & 0.16 & 0.23 \\
\hline & Avg. & 0.83 & 0.79 & 0.78 & 0.19 & 0.20 & 0.20 \\
\hline \multirow[t]{5}{*}{ SHPD 10} & 11 & 0.70 & 0.95 & 0.73 & 0.30 & 0.07 & 0.29 \\
\hline & 12 & 0.87 & 0.88 & 0.90 & 0.18 & 0.23 & 0.10 \\
\hline & 13 & 0.76 & 0.90 & 0.80 & 0.33 & 0.09 & 0.27 \\
\hline & 14 & 0.96 & 0.93 & 0.89 & 0.24 & 0.22 & 0.11 \\
\hline & Avg. & 0.84 & 0.91 & 0.83 & 0.27 & 0.15 & 0.19 \\
\hline
\end{tabular}

amount of cooperation attempts in the first rounds of a sequence. In line with the previous finding that first-round cooperation rates are higher in stage 2 as compared with stage 1, we observe that best response rates are higher in stage 2 than in stage 1 as well.

When the games are more similar as in PDSH 10 and SHPD 10 best response rates are quite similar across the PD's and SH's. More precisely, best response rates in the PD's range from 0.6 to 0.95 and in the SH's they range from 0.66 to 0.96 and are more dispersed than in PDSH 20 and SHPD 20 in both cases. ${ }^{10}$ This indicates that risk dominance not only has an impact on the formation of beliefs, i.e., less cooperation is expected in the $\mathrm{SH}, T=15$ than in the SH, $T=10$, but that it also has an effect on individuals' best response behavior.

At first glance, it is striking how close beliefs are on average to actual behavior in each stage in PDSH 20 and SHPD 20, but not so when the two games are more similar as in PDSH 10 and SHPD 10 (see Table 3). In PDSH 10 subjects tend to be too optimistic about cooperation rates in the stage 1, PD (see column 7 and 10, Table 3), with little learning or adjustment over time. By contrast, subjects are too pessimistic about cooperation rates

\footnotetext{
${ }^{10}$ Note that low best response rates in the $\mathrm{SH}, T=15$ are not due to the two groups coordinating on the inefficient all- $Y$ equilibrium. Rather, it is the case that best response rates are in general lower in the $\mathrm{SH}$, $T=15$ than in the $\mathrm{SH}, T=10$.
} 
in the stage 2, SH game as they expect too little cooperation on average. ${ }^{11}$ In $S H P D 10$, subjects' beliefs initially seem to be well calibrated to actual behavior in the stage $1, \mathrm{SH}$, on average. But again, in the stage 2, PD game they expect too much cooperation and in the stage 3 , SH game too little cooperation.

The last three columns in Table 5 provide a more detailed look at how well individual beliefs are calibrated to opponents' actual behavior at the individual level. Recall that subjects had to indicate how many other subjects in their group will choose $X$. While this is an estimate about the frequency of cooperation in the population, we take it here as the probability with which the current opponent will cooperate. In order to assess the accuracy of the stated belief, we calculate the mean squared deviation, which is defined as $1 / N \sum\left(b_{i}-a_{j}\right)^{2}$ where $b_{i}$ is individual $i$ 's stated belief and $a_{j}$ is the opponents' action, which is either $1(X)$ or $0(Y)$. Notice that this measure is equivalent to a quadratic scoring rule.

From Table 5 it is apparent that the accuracy of subjects' beliefs reflects the observed variation of choices in the two treatments. For example, it confirms that there is little scope for miscalibration in $\mathrm{SH}, T=10$ where play immediately reaches the cooperative outcome and is stable throughout the stages. The mean squared deviations in $\mathrm{SH}, T=10$ are between 0 and 0.14 . Unsurprisingly, beliefs are more likely miscalibrated in the PD's where the mean squared deviations range between 0.08 and 0.22 for $\mathrm{PD}, T=30$ and between 0.07 and 0.30 for $\mathrm{PD}, T=25$. Miscalibrations of a similar magnitude can be observed for the $\mathrm{SH}, T=15$ game in which the mean squared deviations range between 0.10 and 0.33 . To put these numbers in perspective, notice that assigning roughly equal probabilities to the two actions would result in a squared deviation of 0.20 (if more weight -5 out of 9 others - is placed on the actual choice) and 0.31 (if less weight -4 out of 9 others - is placed on the actual choice). ${ }^{12}$ Taking a squared deviation of 0.20 as a benchmark, the accuracy of stated beliefs is significantly higher for the $\mathrm{PD}, T=30$ game and the $\mathrm{SH}, T=10$, game but not when the two games are more similar $(\mathrm{PD}, T=25$ and $\mathrm{SH}, T=15) .{ }^{13}$ The lower accuracy of beliefs when the games are more similar suggests that the lower (higher) temptation to deviate from cooperation in the PD (SH) creates greater strategic uncertainty about opponents' behavior. This increase in strategic uncertainty may also explain why we observe lower best response rates in PDSH 10 and SHPD 10.

\subsection{Cooperation at the individual level}

In order to get a better sense of what determines the observed behavioral patterns in the treatments, we now turn to an analysis of individual behavior. Table 6 shows the frequency of

\footnotetext{
${ }^{11}$ This could be due to low cooperation rates in the stage 1 , PD game. In fact, this pessimism occurs in two sessions with a low cooperation rate in stage 1 . In stage 3, eventually, subjects' expectations about cooperation rates in the $\mathrm{PD}$ are closer to actual behavior in the PD.

${ }^{12}$ It is technically not possible to report equal probabilities, since subjects had to indicate how many of the other nine group members would choose cooperation $(X)$.

${ }^{13} \mathrm{~A}$ Wilcoxon signed-rank test comparing the mean squared deviations of all games with the same parameters with the benchmark of 0.20 yield the following p-values: $p=0.046(\mathrm{PD}, T=30), p=0.027(\mathrm{SH}$, $T=10), p=0.58(\mathrm{PD}, T=25)$ and $p=0.34(\mathrm{SH}, T=15)$.
} 


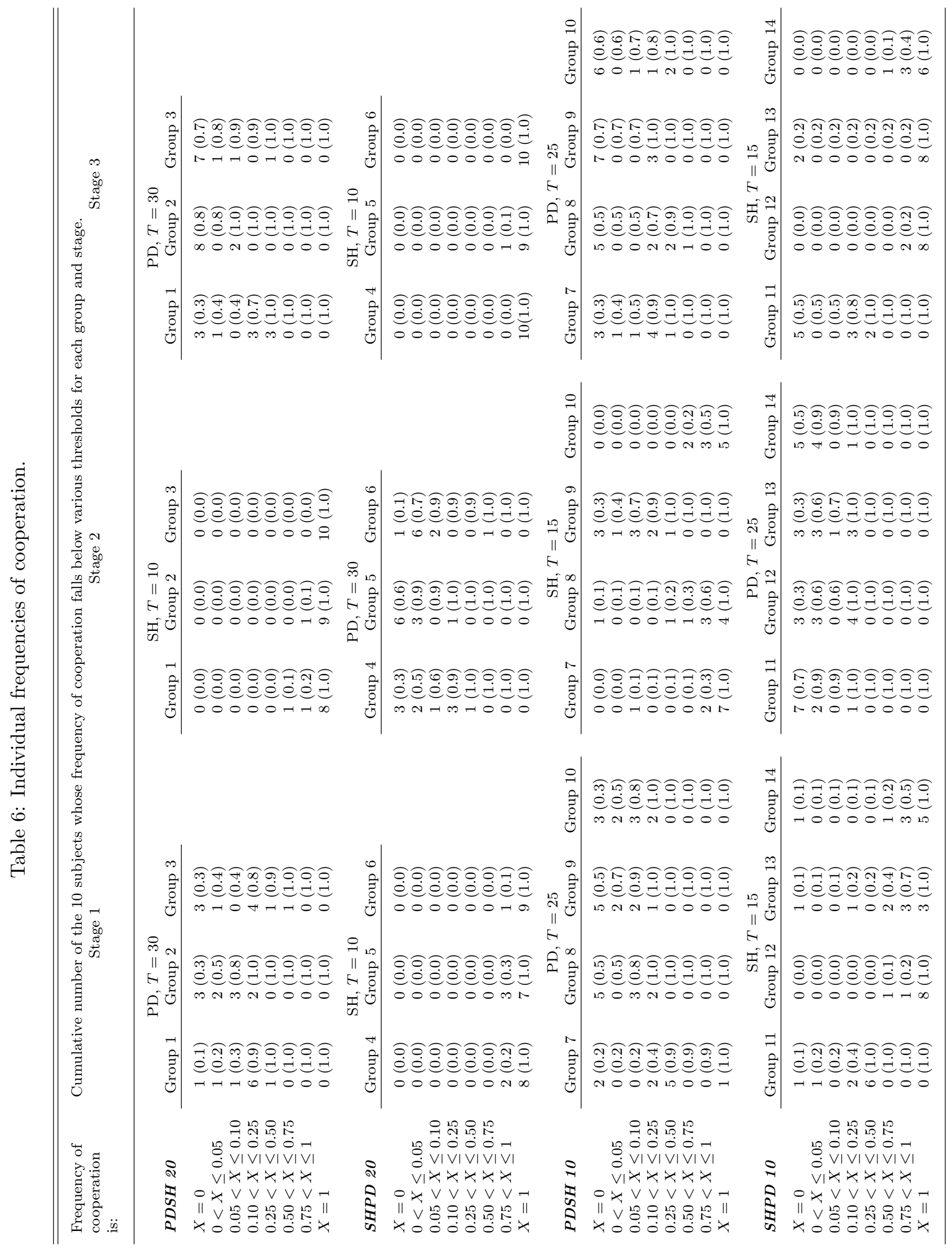


cooperation at the individual level. More precisely, Table 6 reports the number of subjects (cumulative frequencies) whose cooperation rates fall into one of eight ranges, using data from each stage separately.

The table reveals some heterogeneity of individual behavior in the two games. For example, in group 3 of the PDSH 20 treatment, four subjects cooperated in 10 to 25 percent of all rounds and one subject cooperated even in 50 to 75 percent of all rounds in the stage 1 , PD. In other groups the cooperation rates in PD's are often below 10 percent in a stage and most of the cooperation happens in the first few rounds of a stage, as shown in Figure 1 and 2 .

Cooperation is of course more prevalent in the SH's, as shown in Figures 1 and 2 or Table 4. For example, in SH, $T=10$ only one subject cooperates in less than 75 percent of rounds and the great majority of other subjects cooperate in all rounds of a stage. From the previously shown evidence, we know that cooperation rates are clearly affected by risk dominance considerations. In $\mathrm{SH}, T=15$, where the choice of $Y$ is risk dominant, we observe less play of $X$ and cooperation frequencies below 50 percent are more common. For example, we see two groups in which no subject cooperated in more than 50 percent of rounds within a stage.

Table 6 also reveals that some subjects never changed their action. That is, they always play $Y$ in PD's or they always play $X$ in SH's. On average, about 28 percent (41 percent) of subjects never change their action $Y$ in the $\mathrm{PD}, T=30(\mathrm{PD}, T=25)$, whereas 85 percent (40 percent) stick to $X$ throughout a stage in $\mathrm{SH}, T=10(\mathrm{SH}, T=15)$. In addition, in $\mathrm{SH}$, $T=15$ a few subjects ( 9 percent) always play $Y$, which seems to influence the frequency of switching choices in this game. It appears that some subjects cannot be dissuaded from playing a particular action and they stick to their choice throughout a stage. This inertia in choice behavior is more common in less similar games (PDSH 20 and SHPD 20) than in more similar games (PDSH 10 and SHPD 10).

Further details on individual choices is provided by the regressions in Table 7, which shows estimates for the probability of choosing $X$ in each stage. ${ }^{14}$ For each stage we pool the data of the same type of game and include a dummy variable to control for differences in $T$. For stage 1, for example, we run one regression using data of the stage $1, \mathrm{PD} T=30$ and stage $1, \mathrm{PD} T=25$ and one regression using data of the stage $1, \mathrm{SH} T=10$ and stage 1 , SH $T=15$. We concentrate on rounds $1-21$ in a stage, since most of the changes in action choices happened in this initial period (as shown in Figures 1 and 2). Our main interest in these regressions is how an opponent playing $Y$ influences an individuals' choice in the following round. To this end we include a dummy variable taking a value of one if the opponent in the previous round has played $Y$. We also include an interaction term to capture differential effects of opponents' $Y$ choice in the two treatments. Simultaneously, we control for an individuals' own choice in the previous period. In the regression for stages 2 and 3 , we additionally include a variable which controls for the level of cooperation within the matching group in the previous stage (i.e., stage 1 in the stage 2 regressions and stage 2

\footnotetext{
${ }^{14}$ We report here the results of linear probability regressions, because the interpretation of marginal effects from interaction terms in non-linear models is problematic (Ai and Norton, 2003).
} 
Table 7: Regression: determinants of individual choice within a stage

\begin{tabular}{|c|c|c|c|c|c|c|}
\hline \multirow{2}{*}{$\begin{array}{l}\text { dependent variable: } \\
\text { Choice }=X\end{array}$} & \multicolumn{2}{|c|}{ Stage 1} & \multicolumn{2}{|c|}{ Stage 2} & \multicolumn{2}{|c|}{ Stage 3} \\
\hline & PD & $\mathrm{SH}$ & PD & $\mathrm{SH}$ & PD & SH \\
\hline Round in stage & $\begin{array}{c}-0.006 * * \\
(0.002)\end{array}$ & $\begin{array}{l}0.004 * * \\
(0.002)\end{array}$ & $\begin{array}{r}-0.003 \\
(0.002)\end{array}$ & $\begin{array}{l}0.006 * * \\
(0.002)\end{array}$ & $\begin{array}{c}-0.002 * \\
(0.001)\end{array}$ & $\begin{array}{c}0.001 \\
(0.001)\end{array}$ \\
\hline$T=\{15,25\}$ & $\begin{array}{r}-0.081 \\
(0.077)\end{array}$ & $\begin{array}{c}-0.171 * \\
(0.079)\end{array}$ & $\begin{array}{c}0.071 \\
(0.146)\end{array}$ & $\begin{array}{l}-0.157 * * \\
(0.051)\end{array}$ & $\begin{array}{c}0.007 \\
(0.116)\end{array}$ & $\begin{array}{r}-0.092 \\
(0.055)\end{array}$ \\
\hline Opponents' choice $=Y$ in $t-1$ & $\begin{array}{l}-0.312 * * * \\
(0.044)\end{array}$ & $\begin{array}{l}-0.766 * * * \\
(0.119)\end{array}$ & $\begin{array}{r}-0.098 \\
(0.075)\end{array}$ & $\begin{array}{l}-0.716 * * * \\
(0.065)\end{array}$ & $\begin{array}{r}-0.229 * \\
(0.103)\end{array}$ & $\begin{array}{c}-0.742 * * \\
(0.212)\end{array}$ \\
\hline $\begin{array}{l}\text { Opponents' choice }=Y \text { in } t-1 \\
\times T=\{15,25\}\end{array}$ & $\begin{array}{r}0.099 * \\
(0.050)\end{array}$ & $\begin{array}{c}0.018 \\
(0.123)\end{array}$ & $\begin{array}{r}-0.073 \\
(0.151)\end{array}$ & $\begin{array}{r}-0.056 * \\
(0.028)\end{array}$ & $\begin{array}{c}0.009 \\
(0.118)\end{array}$ & $\begin{array}{r}-0.099 \\
(0.159)\end{array}$ \\
\hline Own choice in $t-1$ & $\begin{array}{l}0.415 * * * \\
(0.082)\end{array}$ & $\begin{array}{l}0.657 * * * \\
(0.123)\end{array}$ & $\begin{array}{l}0.211 * * * \\
(0.045)\end{array}$ & $\begin{array}{l}0.721 * * * \\
(0.083)\end{array}$ & $\begin{array}{l}0.532 * * * \\
(0.037)\end{array}$ & $\begin{array}{l}0.775 * * * \\
(0.168)\end{array}$ \\
\hline $\begin{array}{l}\text { Group cooperation level } \\
\text { in stage } 1\end{array}$ & & & $\begin{array}{c}0.020 \\
(0.015)\end{array}$ & $\begin{array}{c}0.336 \\
(0.262)\end{array}$ & & \\
\hline $\begin{array}{l}\text { Group cooperation level } \\
\text { in stage } 2\end{array}$ & & & & & $\begin{array}{l}0.071 * * * \\
(0.013)\end{array}$ & $\begin{array}{c}0.695 \\
(0.947)\end{array}$ \\
\hline Constant & $\begin{array}{l}0.422 * * * \\
(0.059)\end{array}$ & $\begin{array}{l}0.938 * * * \\
(0.022)\end{array}$ & $\begin{array}{r}0.144 * \\
(0.059)\end{array}$ & $\begin{array}{l}0.886 * * * \\
(0.043)\end{array}$ & $\begin{array}{l}0.206 \\
(0.112)\end{array}$ & $\begin{array}{l}0.931 * * * \\
(0.077)\end{array}$ \\
\hline $\mathrm{N}$ & 1400 & 1360 & 1400 & 1400 & 1170 & 1400 \\
\hline$R^{2}$ & 0.24 & 0.39 & 0.09 & 0.50 & 0.31 & 0.63 \\
\hline
\end{tabular}

Linear Probability regressions using data from rounds 1-21 in each stage with robust standard errors clustered at the matching group level in parentheses. The variable " $T=\{15,25\}$ " is a dummy variable capturing treatment differences between same games. The variable "Opponents' choice $=Y$ in $t-1$ " is a dummy variable which equals one if opponent's choice was $Y$ ("Defect") in the previous round and "Opponents' choice $=Y$ in $t-1 \times T=\{15,25\}$ " is the interaction of these two variables. "Own choice in $t-1$ " is a dummy variable which equals one if own choice was $X$ in the previous round. The variables "Group cooperation level in stage 1" and "Group cooperation level in stage 1" capture the average cooperation level in a matching group in stage 1 , respectively, stage 2 .

in the stage 3 regressions).

First of all, the coefficients for "Round in stage" provides further support for the previous observations that the frequency of cooperation decreases in the PD and increases in the SH as play progresses. Furthermore, the treatment variable confirms that the frequency of cooperation is usually lower in $\mathrm{SH}, T=15$ than in $\mathrm{SH}, T=10$ in the first two stages, whereas in the PD's no differences are observed.

We find that it matters in most cases whether the opponent has played $Y$ in the previous round. In general, this effect is negative, but its size and significance differs across stages and games. Looking first at PD's, we observe that subjects in the stage 1, PD $T=30$ are highly likely to play $Y$ after encountering an opponent playing $Y$ in the previous round. This is also the case for the stage $1, \mathrm{PD} T=25$ but the effect is smaller than in the stage $1, \mathrm{PD}$ $T=30$, as indicated by the positive and significant interaction term. In the stage 2, PD's the impact of opponents play of $Y$ is negative, but not significant for both $\mathrm{PD} T=30$ and 
PD $T=25$. There is some indication that a choice of $X$ in stage 2 is more likely for subjects who experienced a more cooperative environment in the first stage. Albeit the coefficient is not significant at conventional levels. Stage 3 yields a similar pattern as stage 1, that is, we observe a lower likelihood of $X$ after experiencing defection in the previous round. The insignificant interaction term suggests that this is the case in both PD $T=30$ and PD $T=25$. Furthermore, we see that the coefficient for the cooperation level in stage 2 is significant. That is, it is significantly more likely that a subject chose $X$ if she was previously in a more cooperative environment.

Turning to the SH regressions (columns 2, 4 and 6 in Table 7) we find similar patterns as for the PD. Having played against an defecting opponent, has a negative and significant impact in all stages and the effect is sizable. While in stage 1 and 3 there are no treatment differences, as evidenced by the insignificant coefficient for "Opponents' choice $=Y$ in $t-1$ $\times T=\{15,25\}$ ", the interaction term is significant and negative for stage 2 , indicating an overall larger effect in games with $T=\{15,25\}$. That is, subjects are significantly less likely to play $X$ after previously experiencing play of $Y$ in $\mathrm{SH}, T=15$ than in $\mathrm{SH}, T=10$. There is no evidence that previous experience in stage 1 or 2 has an effect on the choice of $X$ in stage 2 and 3.

We conclude that encountering a non-cooperative opponent increases the probability of a switch from cooperative play to defection, which in most cases means defection for the remaining rounds. Moreover, a sizeable fraction of subjects is hesitant to change their actions within stages.

\subsection{What determines the shift in behavior between stages?}

While there is some inertia with respect to actions within stages, we have seen evidence for large swings in behavior between stages. More specifically, when the temptation payoff $T$ is lowered after stage 1, i.e., when the PD turns into a SH, almost every subject switches from playing $Y$ to playing $X$ in treatment $P D S H 20$ (87 percent). This change is less dramatic in $P D S H 10$ where on average only two-thirds of subjects change their action. We can reject the hypothesis of equal switching rates in the first round of stage 2 in $P D S H 20$ and $P D S H 10$ using a robust rank-order test $(p=0.029)$. The same pattern is true when $T$ is lowered after stage 2, which is the case in $S H P D 20$ and $S H P D$ 10. In SHPD 20, 90 percent of subjects switch from $Y$ to $X$, whereas only 65 percent switch in $S H P D$ 10. Again, we can reject the hypothesis of equal switching rates in the first round of stage 3 in SHPD 10 and SHPD 20 (robust rank-order test, $p=0.057$ ).

Interestingly, when the temptation payoff $T$ is increased after stage 1, i.e., a switch from SH to PD, on average 60 percent of subjects in SHPD 20 and 47 percent of subjects in SHPD 10 change their action. According to a robust rank-order test this difference is not statistically significant $(p>0.114)$. When the same change in $T$ occurs after stage 2 , the switching rate is 80 percent in $P D S H 20$ and only 47 percent in $P D S H 10$. Again, the

difference is not statistically significant using a robust rank-order test $(p>0.114)$. Therefore a shift in behavior between stages is more pronounced in games that are less similar as it is 
Table 8: Regression: determinants of choice in first round after a game change

\begin{tabular}{|c|c|c|c|c|c|c|}
\hline \multirow{2}{*}{$\begin{array}{l}\text { dependent variable: } \\
\text { Choice }=X \\
\text { Belief }\end{array}$} & \multicolumn{3}{|c|}{ change from PD to SH } & \multicolumn{3}{|c|}{ change from $\mathrm{SH}$ to $\mathrm{PD}$} \\
\hline & $\begin{array}{l}0.362 * * * \\
(0.085)\end{array}$ & $\begin{array}{l}0.395 * * * \\
(0.094)\end{array}$ & $\begin{array}{l}0.378 * * * \\
(0.089)\end{array}$ & $\begin{array}{l}0.627 * * * \\
(0.111)\end{array}$ & $\begin{array}{l}0.630 * * * \\
(0.136)\end{array}$ & $\begin{array}{l}0.654 * * * \\
(0.107)\end{array}$ \\
\hline Own 1st round choice & $\begin{array}{l}0.148 * * \\
(0.058)\end{array}$ & & $\begin{array}{l}0.182 * * * \\
(0.062)\end{array}$ & $\begin{array}{l}0.263 * * * \\
(0.084)\end{array}$ & & $\begin{array}{l}0.276 * * * \\
(0.078)\end{array}$ \\
\hline Experienced cooperation & & $\begin{array}{c}0.263 \\
(0.319)\end{array}$ & $\begin{array}{l}0.670 * * \\
(0.281)\end{array}$ & & $\begin{array}{c}0.009 \\
(0.077)\end{array}$ & $\begin{array}{c}-0.108 \\
(0.123)\end{array}$ \\
\hline Decision time & $\begin{array}{c}-0.008 \\
(0.006)\end{array}$ & $\begin{array}{r}-0.009 * \\
(0.005)\end{array}$ & $\begin{array}{c}-0.013 * * \\
(0.006)\end{array}$ & $\begin{array}{c}-0.012 * * * \\
(0.005)\end{array}$ & $\begin{array}{c}-0.011 * * \\
(0.004)\end{array}$ & $\begin{array}{c}-0.012 * * * \\
(0.005)\end{array}$ \\
\hline$T=\{15,25\}$ & $\begin{array}{c}-0.103 \\
(0.089)\end{array}$ & $\begin{array}{c}-0.097 \\
(0.097)\end{array}$ & $\begin{array}{c}-0.110 \\
(0.078)\end{array}$ & $\begin{array}{c}0.088 \\
(0.079)\end{array}$ & $\begin{array}{c}0.058 \\
(0.095)\end{array}$ & $\begin{array}{c}0.062 \\
(0.084)\end{array}$ \\
\hline $\mathrm{N}$ & 120 & 120 & 120 & 120 & 120 & 120 \\
\hline $\begin{array}{l}* p<0.10,{ }^{* *} p<0.05,{ }^{* *} \\
\text { Probit regressions (averas } \\
\text { level in parentheses. "Be } \\
\text { "Own 1st round cooperat } \\
\text { and "Experienced Coop } \\
\text { "Decision time" is the ti }\end{array}$ & $\begin{array}{l}p<0.01 \\
\text { marginal ef } \\
\text { f" is the el } \\
\text { n" indicate } \\
\text { tion" is th } \\
\text { e needed fo }\end{array}$ & $\begin{array}{l}\text { ects) with rol } \\
\text { cited belief ir } \\
\text { whether a st } \\
\text { average coc } \\
\text { a decision i }\end{array}$ & $\begin{array}{l}\text { bust standarc } \\
n \text { the first ro } \\
\text { ubject cooper } \\
\text { operation of } \\
\text { in seconds. }\end{array}$ & $\begin{array}{l}\text { errors clust } \\
\text { nd of a sta\& } \\
\text { ated in first } \\
\text { ne's oppon } \\
\Gamma=\{15,25\end{array}$ & $\begin{array}{l}\text { ered at the } \\
\text { ge and is b } \\
\text { round of th } \\
\text { gent in the } \\
\text { 6" is an in }\end{array}$ & $\begin{array}{l}\text { ching group } \\
\text { en } 0 \text { and } 1 . \\
\text { rst sequence } \\
\text { vious stage. } \\
\text { tor variable }\end{array}$ \\
\hline
\end{tabular}

the case in PDSH 20 and SHPD 20. Recall that Hypothesis 2 implicitly assumes that changes in behavior between stages are smaller when the games are more similar. The finding that the observed swings in behavior between stages are less pronounced in the more similar games provides some qualitative support for Hypothesis 2.

What factors can account for these large and sudden swings of behavior in the first round of a stage? Evidence can be found in Table 8, which reports the results of several probit regressions with a subject's choice of $X$ in the first round of a stage as the dependent variable. The first three columns in Table 8 focus on situations in which the game changes from a PD to a SH. Notice that this situation occurs either at the beginning of stage 2 (in PDSH 20 and $P D S H 10$ ) or at the beginning of stage 3 (in SHPD 20 and SHPD 10). The regressions pool the data from both situations. Our main variables of interest are the elicited beliefs of subjects and two variables capturing past behavior: a subject's choice in the first round of the first sequence and a subject's experience with cooperation among all opponents, i.e., the average cooperation rate of subjects' opponents in the previous stage. Additionally, we include the decision time (in seconds) and an indicator variable $T=\{15,25\}$ to capture the differences between treatments.

It is apparent that beliefs are important: expecting more opponents to choose cooperative play is positively correlated with play of the cooperative action $X$. The coefficient on "Belief" is significantly positive and of a similar size in all three regressions looking at the transition from the PD to the $\mathrm{SH}$. A second important factor is whether a subject cooperated in the 
first round of the session. These subjects are significantly more likely to choose $X$ in the first round of a game change. Experience, on the other hand, seems to play only a minor role. Although an exposure to "more cooperative" opponents is positively associated with cooperation in the first round of stage 2 or 3 , it is only significant when a subject's first round choice is included in the regression, suggesting that cooperation experience is only important for first-round cooperators. Furthermore, faster decisions, as measured by decision time, more likely result in cooperation, while defection appears to be a more deliberate decision. The size of this decision time effect, however, is small and the statistical significance of it depends on which covariates are included in the regression. Perhaps most importantly, after controlling for the other independent variables we observe no treatment effect in all three specifications as evidenced by the insignificant coefficient on the $T$ variable.

The regressions in the last three columns in Table 8 consider situations in which the game changes from a SH to a PD. That is the case at the beginning of stage 2 (in SHPD 20 and $S H P D$ 10) and at the beginning of stage 3 (in PDSH 20 and $P D S H$ 10). The regressions include the same set of independent variables as in the previous set of regressions. The results are largely the same as before except that the impact of experienced cooperation is now smaller than before and, more importantly, is never significant. This finding is not so surprising as subjects typically experience a high level of cooperation throughout the preceding SH's and thus the variation of play is usually lower than in the PD's. Notice that the coefficient estimates for "Beliefs" as well as for "Own 1st round choice" are large and significant suggesting an even stronger association than for the transition from the PD to the SH. Finally, we again observe a negative impact of the decision time on the choice of $X$, suggesting that some subjects may speculate that others are cooperators and deliberate about whether to exploit these cooperators.

As a final piece of evidence, we analyze how subjects' beliefs change when they encounter a particular game for the first time. That is, we are interested in the change between initial beliefs and beliefs in the first round of a change in $T$. In particular, we concentrate here on two factors that may contribute to a change in beliefs and that are independent of a subject's experience within a stage: a subject's initial propensity to cooperate and the initial frequency of cooperative encounters in the first stage. Let $b_{1 s}$ denote a subjects' belief about the number of others playing $X$ in round 1 of stage $s=1,2$ and denote the difference in such beliefs by $\Delta b=b_{12}-b_{11}$.

First, Table 3 (columns 7 and 8) indicates large swings in beliefs. For example, subjects expect less cooperation by others after an increase of $T$ as $\triangle b=-0.39$ in $S H P D 20$ and $\Delta b=-0.34$ in $S H P D$ 10. By contrast, subjects expect more cooperation from others after a decrease in $T$ as in PDSH $20(\Delta b=0.33)$ but not in PDSH $10(\Delta b=-0.05) .{ }^{15}$ More important, though, is the significantly negative correlation between $\Delta b$ and first-round choices in all treatments except PDSH $10{ }^{16}$ In fact, first-round defectors adapt their beliefs

\footnotetext{
${ }^{15}$ There is little correlation between initial beliefs $b_{11}$ and first-round second-stage beliefs $b_{12}$ except when $\Delta b$ is small such as in $P D S H$ 10. The correlation coefficients for $b_{11}$ and $b_{12}$ are $0.20(p=0.39)$ in $P D S H 20$, $0.33(p<0.04)$ in PDSH 10, -0.21 $(p=0.39)$ in SHPD 20 and $-0.15(p=0.37)$ in SHPD 10.

${ }^{16}$ The correlation coefficient in PDSH 10 is $-0.08(p=0.62)$, whereas coefficients range between -0.54 and
} 
more drastically than cooperators in the transition from the PD to the $\mathrm{SH}$, while the opposite holds for the transition from SH to PD. This suggests that those who initially chose the majority action within a stage ( $Y$ in PD's and $X$ in SH's) are more sensitive to the strategic incentives of the games.

Second, to examine the impact of initial experience on subjects' changing beliefs we consider the role of their opponents' play in the first two rounds of stage 1 . Notice that because of our random-matching procedure opponents' behavior in the first two rounds is not correlated with the past behavior of a subject. We expect that $\Delta b$ is greater for subjects who experienced more cooperation in the first two rounds than subjects who were confronted with less cooperation. Indeed, this is what we find for all treatments. However, the correlation is significant only for treatments PDSH 10 and SHPD 10. ${ }^{17}$ That is, experiencing more initial cooperation leads on average to a smaller change in beliefs when the games are more similar as in PDSH 10 and SHPD 10.

Summarizing the discussion of beliefs, we find evidence that subjects' choices in the first round influence the updating of their beliefs when the games change. Further, experienced cooperation from opponents also plays a role in this belief updating but only when the games are more similar.

\section{Conclusion}

We have conducted and reported on an experiment examining behavior and equilibrium selection in two similar, infinitely repeated games, Stag Hunt and Prisoner's Dilemma under anonymous random matching. Our aim was to understand the role of historical precedents for equilibrium selection by varying the similarity across the two games. Arguably, the similarity of two games is an important consideration for effective transfers of precedent. For example, Fudenberg and Kreps (1988) noted “..., as seems reasonable, players infer about how their opponents will act in one situation from how opponents acted in other, similar situations."

Our main finding is that the role of precedent for equilibrium selection in the repeated games that we study appears to be rather limited; the history of play in different but similar games appears to have little impact on behavior. Following small changes in the value of the temptation payoff parameter, there are large swings in the frequency with which players play the two actions available to them. More precisely, the frequency of play of the cooperative action is stochastically larger in SH parameterizations of the stage game than in PD parameterizations of the stage game, regardless of the order in which these two types of games are played and despite the fact that both pure equilibria of the $\mathrm{SH}$ can also be supported as equilibria under the PD using the contagious, society-wide grim trigger strategies of Kandori (1992). There is, however, some variation in the frequency of the cooperative action in games that are more similar. For example, there are instances where a

-0.56 in PDSH 20, SHPD 20 and SHPD 10 (p-values $<0.015$ ).

${ }^{17}$ The correlation coefficients are $0.14(p=0.54)$ in PDSH 20, $0.04(p<0.87)$ in $S H P D 20,0.31(p=0.054)$ in PDSH 10 and $0.35(p=0.026)$ in SHPD 10. 
precedent of coordination on the inefficient pure equilibrium of the Stag Hunt game carries over to the subsequent Prisoner's dilemma game or the reverse transfer occurs, but such transfer of precedent is not the norm, and only occurs when the games are more similar to one another. Our results suggest that strategic considerations guide behavior in our indefinitely repeated games and that there appears to be little use of equilibrium selection precedents across similar games.

There are several possible directions in which our analysis of the role of precedent in repeated games might be profitably extended. First, one could consider changes in the value of $T$ that are in an even smaller neighborhood of the threshold value that differentiates the $\mathrm{SH}$ from the PD to examine whether such smaller changes might give precedents even more weight. In addition, one might explore the role of precedent across similar games played in continuous time; Friedman and Oprea (2012), for instance, report varying but very high cooperation rates for different parameterizations of the Prisoner's Dilemma game played by subjects in continuous time. It would be of interest to consider play in continuous (or near continuous) time across similar classes of games including the Stag Hunt and Prisoner's Dilemma games or the Battle of the Sexes and Chicken games. Finally, we would ultimately like to endogenize the transition between games, as is done in stochastic (Markov) games, or endogenize the value of the temptation parameter, $T$ itself. $^{18}$ Specifically, one might suppose that temptation, $T$, was a steadily increasing function of the time that players spent playing the efficient, $X X$ outcome and, symmetrically, the temptation value $T$ was a steadily decreasing function of the time that players spent playing the inefficient $Y Y$ outcome. In such a setting it would be possible to imagine cycles of coordinated play between the efficient and inefficient outcomes with transitions occurring endogenously according to some threshold value for $T$. We leave such extensions to future research.

\section{References}

Ahn,T.K., Ostrom Elinor, Schmidt David, Shupp Robert and James Walker (2001), "Cooperation in PD Games: Fear, Greed and History of Play," Public Choice 106, 137-155.

Ai, Chunrong and Norton, Edward C. (2003), "Interaction Terms in Logit and Probit Models," Economics Letters 80, 123129.

Brandts, Jordi and Cooper, David J. (2006), "A Change Would Do You Good: An Experimental Study on How to Overcome Coordination Failure in Organizations," American Economic Review 96, 669-693.

Bohnet, Iris and Steffen Huck (2004), "Repetition and Reputation: Implications for Trust

\footnotetext{
${ }^{18}$ Vespa and Wilson (in preparation) have studied Markov games in the laboratory. Dal Bò, Dal Bò and Eyster (2013) report on an experiment where subjects endogenously choose, via a majority rule vote, whether to participate in a Prisoner's dilemma game or an alternative (anti-PD game) where cooperation is a dominant strategy; they find that a slight majority (53.6\%) vote for the PD game!
} 
and Trustworthiness When Institutions Change," American Economic Review 94, 362366.

Camera, Gabriele, and Marco Casari (2009), "Cooperation Among Strangers Under the Shadow of the Future," American Economic Review 99, 979-1005.

Camerer, Colin F. (2003) Behavioral Game Theory, Princeton: Princeton University Press.

Cason, Timothy, Savikhin Anya and Roman M. Sheremeta (2012), "Behavioral Spillovers in Coordination Games," European Economic Review 56, 233-245.

Dal Bò, Ernesto, Pedro Dal Bò and Erik Eyster (2013), "The Demand for Bad Policy When Voters Underappreciate Equilibrium Effects," working paper, Brown University.

Dal Bò, Pedro, and Guilleaume Frechette (2011), "The Evolution of Cooperation in Infinitely Repeated Games: Experimental Evidence," American Economic Review 101, 411-429.

Devetag, Giovanna (2005), "Precedent Transfer in Coordination Games: An Experiment," Economics Letters 89, 227-232.

Duffy, John and Jack Ochs (2009), "Cooperative Behavior and the Frequency of Social Interaction," Games and Economic Behavior 66, 785-812.

Dufwenberg, Martin, Sundaram, Ramya and David J. Butler (2010), "Epiphany in the Game of 21," Journal of Economic Behavior and Organization 75, 132-143.

Feltovich, Nick (2003), "Nonparametric Tests of Differences in Medians: Comparison of the WilcoxonMannWhitney and Robust Rank-Order Tests," Experimental Economics 6, 273-297.

Fischbacher, Urs (2007), “z-Tree: Zurich Toolbox for Ready-Made Economic Experiments," Experimental Economics 10, 171-178.

Friedman, Daniel and Ryan Oprea (2012), "A Continuous Dilemma," American Economic Review, 102, 337-363.

Fudenberg, Drew and David M. Kreps (1988), "A Theory of Learning, Experimentation, and Equilibrium in Games," working paper, MIT.

Kandori, Michihiro (1992), "Social Norms and Community Enforcement," Review of Economic Studies, 59, 63-80.

Knez, Marc (1998), "Precedent transfer in Experimental Conflict-of-Interest Games," Journal of Economic Behavior and Organization 34, 239-249. 
Knez, Marc and Colin F. Camerer (2000), "Increasing Cooperation in Prisoner's Dilemmas by Establishing a Precedent of Efficiency in Coordination Games," Organizational Behavior and Human Decision Processes 82, 194-216.

Lucas, Robert E. Jr. (1986), "Adaptive Behavior and Economic Theory," Journal of Business 59, S401-426.

Ochs, Jack (1995), "Coordination Problems," in J.H. Kagel and A.E Roth, Eds., The Handbook of Experimental Economics Princeton: Princeton University Press, pp. 195251.

Peysakhovich, Alexander and David G. Rand (2013), "Habits of Virtue: Creating Norms of Cooperation and Defection in the Laboratory," working paper, Harvard and Yale Universities.

Rankin, Frederick W., John B. Van Huyck, and Raymond C. Battalio (2000), Strategic Similarity and Emergent Conventions: Evidence from Similar Stag Hunt Games," Games and Economic Behavior 32, 315-337.

Roth, Alvin E. and J. Keith Murnighan (1978), "Equilibrium Behavior and Repeated Play of the Prisoner's Dilemma," Journal of Mathematical Psychology 17, 189-198.

Van Huyck, John B., Raymond C. Battalio, and Richard Beil (1991), "Strategic Uncertainty, Equilibrium Selection Principles, and Coordination Failure in Average Opinion Games," Quarterly Journal of Economics 106, 885-910.

Vespa, Emanuel and Alistair J. Wilson (2014), "Dynamic Games and Markov Perfection: Putting the 'Conditional' in Cooperation," in preparation.

Weber, Roberto (2006), "Managing Growth to Achieve Efficient Coordination in Larger Groups," American Economic Review 96, 114-126. 


\section{Appendix A}

In the paper we assert that for the parameterizations of the prisoner's dilemma (PD) stage game that we study in the experiment and given our induced discount factor, it is possible to support play of the efficient $X X$ outcome as a sequential equilibrium of the indefinitely repeated $\mathrm{PD}$. In this appendix we explain how we verified this claim following the logic of the contagious sequential equilibrium construction of Kandori (1992).

Consider the $2 \times 2$ game given in Table 1 , where action $X$ corresponds to cooperation and action $Y$ corresponds to defection. Denote the period discount factor (equivalently the continuation probability) by $\delta$ and the population or matching group size by $M$. In our experiment as in one of the environments studied by Kandori, these $M$ players are randomly and anonymously matched in each period of the infinitely repeated game and can only draw upon their own personal histories in the previous $t=0,1,2, \ldots$ matches of the game; player $i$ has no knowledge of the identities of any player nor of what transpires in any matches other than his own and no communication with other players is allowed. Following Kandori (1992), assume that there are just two types of players. All players start off as c-types, playing the cooperative strategy. A player remains a c-type if he has never experienced a defection, but permanently becomes a d-type if he initiates or experiences and defection. The sequential equilibrium associated with this "contagious strategy" is characterized by the type-dependent strategy wherein c-types play cooperate (action $X$ ) and d-types play defect (action $Y$ ). Thus, trust is attached to the society as a whole. A single defection triggers a contagious wave of defection until the economy arrives at the outcome of mutual defection by all $\mathrm{M}$ players. The threat of such a contagion may deter defection from occurring and thus enable play of the efficient, cooperative strategy by all $\mathrm{M}$ players in every period.

Let $D_{t}$ denote the total number of d-type players as of period $t$ and let $A$ denote an $M \times M$ transition matrix with elements $a_{i j}=\operatorname{Pr}\left[D_{t+1}=j \mid D_{t}=i\right]$. Let $B$ denote another $M \times M$ matrix with elements $b_{i j}=\operatorname{Pr}\left[D_{t+1}=j \mid D_{t}=i\right.$ and one d-type player deviates to cooperation (plays $X$ ) at time $t$.]. Denote the conditional probability that a d-type player randomly meets a c-type player when there are already $i$ d-type players as the $i$ th element of the column vector

$$
\rho=\frac{1}{M-1}[M-1, M-2, \ldots, 1,0]^{\prime} .
$$

Finally, let $e_{i}$ denote a $1 \times M$ row vector with $i$ th element equal to 1 and all other elements equal to 0 . Kandori's lemma, that the contagious strategy constitutes a sequential equilibrium, requires that two conditions be satisfied. The first condition, adapted to the game of Table 1 is that

$$
\frac{10}{1-\delta} \geq \sum_{t=0}^{\infty} \delta^{t} e_{1} A^{t} \rho(T-10)=e_{1}(I-\delta A)^{-1}(T-10) .
$$

Here, all payoffs have been normalized relative to the mutual defection payoff of 10 . This condition states that the present value of the normalized payoff from playing the cooperative strategy, (left hand side), equals or exceeds the present value of the normalized payoff from 
defecting in every period (right hand side), where $e_{1} A^{t} \rho$ denotes the probability of meeting a c-type player given that the player initiated a contagious wave of defection at time 0 . This condition can be rewritten as:

$$
\frac{10}{T-10} \geq(1-\delta) e_{1}(I-\delta A)^{-1}
$$

A second condition is that once a contagious wave of defection has been initiated, no d-type player has an incentive to revert to play of the cooperative strategy in an effort to slow down the contagious process. This second condition, adapted to the game of Table 1 , is that a d-type player finds a one-shot deviation to cooperation unprofitable given that $D_{t}=k$, for all $k=2,3, \ldots M$ :

$$
\sum_{t=0}^{\infty} \delta^{t}\left[e_{k} A^{t} \rho(T-10)\right] \geq\left(\frac{M-k}{M-1}-\frac{k-1}{M-1}\right)(10)+\delta \sum_{t=0}^{\infty} \delta^{t}\left[e_{k} B A^{t} \rho(T-10)\right] .
$$

This condition states that the present normalized value from defecting forever when there are $k$ d-type players (left hand side) is greater than the present normalized payoff from a one time deviation to the cooperative strategy by the d-type player who then reverts to playing the defection strategy forever afterward. Note that $\frac{M-k}{M-1}$ is the probability the deviating $\mathrm{d}-$ type player meets a c-type player, $\frac{k-1}{M-1}$ is the probability the deviating d-type player meets a d-type player and $e_{k} B$ represents the distribution of d-type players in the next period given that in the current period there are $k$ d-type players and the one d-type, the candidate player, deviates to the cooperative strategy in the current period.

Using our parameterization of the indefinitely repeated $\mathrm{PD}$, where $T \in\{25,30\}, M=10$ and $\delta=5 / 6$, we have verified numerically that conditions (1) and (2) are always satisfied. ${ }^{19}$ It follows that a social norm of mutual cooperation in all periods comprises a sequential equilibrium of both versions of the infinitely repeated PD that we study in our experiment. Of course, many other equilibria are also possible, including mutual defection in all periods.

\footnotetext{
${ }^{19}$ The Mathematica program we used to verify these conditions is available upon request.
} 


\section{Appendix B}

\section{Instructions used in PDSH 20 and SHPD 20. (Instructions for $P D S H 10$ and SHPD 10 are identical except for different values for $\mathrm{T})$.}

\section{Instructions}

You are about to participate in an economic decision-making experiment. Please read these instructions carefully, as the amount of money you earn may depend on how well you understand these instructions. If you have a question at any time, please feel free to ask the experimenter. We ask that you not talk with the other participants once the experiment has begun.

\section{Overview}

There are 20 participants in todays session. The computer program will begin by randomly dividing you up into two groups of size 10. Your assignment to a group of size 10 will not change for the duration of the experiment. You will only interact with members of your own group of size 10 for the entire experiment.

This experimental session is made up of a number of sequences. Each sequence consists of an indefinite number of rounds. In each round you will be randomly and anonymously matched with one of the other 9 participants in you group of size 10 and asked to make a decision. All matchings with the other 9 participants are equally likely, that is, in each round you have a $1 / 9$ chance of being matched with any single participant in your group of size 10 .

At the beginning of the first sequence, you will be assigned an ID number which is used only for record identification purposes. You will never be told the ID number or the identity of the person you are matched with in any round, nor will he/she ever be told your identityeven after the end of the session.

In each round, both you and the other participant matched to you must choose between two possible decisions labelled $\mathrm{X}$ and $\mathrm{Y}$. You make your decision without knowing the decision of the other participant. You simply click on the radio button next to your decision and then click the OK button. You can change your mind anytime prior to clicking the OK button. After all participants have made their decisions, you will be informed of the decision made by the participant matched to you, and likewise, that other participant will learn of your decision.

Your decision, together with the decision of the participant matched to you results in one of four possible outcomes: X,X, X,Y, Y,X and Y,Y, where the first letter refers to your decision and the second letter refers to the decision of the participant matched to you. The outcome determines your payoff for the round as well as the payoff of the participant matched to you for that round. There are two possible ways in which the four outcomes translate into payoffs, which we refer to as payoff table 1 and payoff table 2 . These are shown below. 


\begin{tabular}{|l|c|c|}
\hline Table 1 & Other's decision: $X$ & Other's decision: $Y$ \\
\hline Your decision $X$ & $\begin{array}{c}\text { Your payoff: } 20 \\
\text { Other's payoff: } 20\end{array}$ & $\begin{array}{c}\text { Your payoff:0 } \\
\text { Other's payoff: } 30\end{array}$ \\
\hline Your decision $Y$ & $\begin{array}{c}\text { Your payoff: } 30 \\
\text { Other's payoff: } 0\end{array}$ & $\begin{array}{c}\text { Your payoff:10 } \\
\text { Other's payoff: } 10\end{array}$ \\
\hline
\end{tabular}

\begin{tabular}{|l|c|c|}
\hline Table 2 & Other's decision: $X$ & Other's decision: $Y$ \\
\hline Your decision $X$ & $\begin{array}{c}\text { Your payoff: } 20 \\
\text { Other's payoff: } 20\end{array}$ & $\begin{array}{c}\text { Your payoff:0 } \\
\text { Other's payoff: } 10\end{array}$ \\
\hline Your decision $Y$ & $\begin{array}{c}\text { Your payoff: 10 } \\
\text { Other's payoff: 0 }\end{array}$ & $\begin{array}{c}\text { Your payoff:10 } \\
\text { Other's payoff: 10 }\end{array}$ \\
\hline
\end{tabular}

Notice that the only difference between payoff table 1 and payoff table 2 is the payoff that you receive in the outcome where you choose $\mathrm{Y}$ and the other participant chooses $\mathrm{X}$, or symmetrically, the payoff the other participant receives when you choose $\mathrm{X}$ and the other participant chooses Y. In all other cases the payoffs are unchanged across the two payoff tables.

\section{Which table?}

Only one payoff table, either Table 1 or Table 2 will be in effect for all members of your group of size 10 and for all rounds of a given sequence. This table will be shown on every participants computer screen prior to their making a decision. Specifically, you and the other 9 participants with whom you can be randomly matched in each round will see the exact same table, which will be clearly labelled as either Table 1 or Table 2 in the upper left hand corner. For further ease of reference, Table 1 will be displayed to all participants in a black color while Table 2 will be displayed to all participants in a red color. The table shown in each round will be the one used to determine your payoffs for that round.

At the start of each new sequence, the payoff table for all 10 members of your group may or may not change from the one used in the previous sequence. Therefore, at the start of each new sequence you will want to carefully examine the payoff table that is being used to determine payoffs in each round of the new sequence. If a change in payoff tables does occur, the change will only occur at the start of a new sequence of rounds; the payoff table shown in the first round of each new sequence will remain the same one in effect for all participants for all subsequent rounds of that sequence. The payoff table may or may not change again at the start of a new sequence.

\section{When does a sequence of rounds end?}

Each sequence consists of at least one round. Following the first round, and every subsequent round, a six-sided die will be rolled. (Participants will take turns rolling the die). If the die 
roll results in a $1,2,3,4$, or 5 being rolled, then the current sequence continues on with another round. If the die roll results in a 6 being rolled, then the current sequence is over. Depending on the amount of time remaining in the session, a new sequence of rounds may then begin. For the new sequence, the table used to determine payoffs may or may not change from the table used in the prior sequence of rounds. Thus, there is a $5 / 6$ chance that a given sequence will continue from one round to the next and a 1/6 chance that the sequence will not continue beyond the current round. Each new sequence will consist of an indefinite number of rounds. In each round of the new sequence you will be randomly matched with one of the other 9 participants as before.

\section{Expectations at the start of each new sequence}

In the first round of each new sequence the computer program will ask you How many of the other 9 participants (excluding you) in your group will choose $\mathrm{X}$ in this round? Please enter your expectation, a number between 0 and 9 inclusive, in the blue input box on your screen. When you have entered this number and made an action choice of $\mathrm{X}$ or $\mathrm{Y}$ for the first round, click the OK button. You will only be asked for this expectation of the number of others choosing $\mathrm{X}$ in the first round of each new sequence.

\section{Results, Record Sheets and your History of Play}

At the end of each round you see a results screen reporting on your decision, the decision of the other participant matched to you, your payoff and the other participants payoff. Please record this information on a new row of your record sheet under the appropriate headings. Then press the OK button to continue. For your convenience, a history of your past results will appear at the bottom of the results screen for ready reference.

\section{Payments:}

Your payoff for each round is a number, either $0,10,20$ or 30. This number represents your payoff for the round in cents, that is, 1 point $=1$ cent, so your possible earnings each round are $\$ 0.00, \$ 0.10, \$ 0.20$ or $\$ 0.30$. At the end of the session you will paid the sum total of your payoff earnings from all rounds of all sequences, plus a payment of $\$ 5$ for completing the session. Payments are made privately and in cash.

\section{Questions?}

Now is the time for questions. If you have a question, please raise your hand and the experimenter will answer your question in private. 


\section{Quiz}

Before we begin, we ask you to complete the following quiz. The purpose of the quiz is to test your understanding of the rules of the experiment. After all participants have completed the quiz, we will analyze the quiz questions. If there are any mistakes, we will go over the relevant part of the instructions again.

Instructions: Circle or write in the blank space the correct answer to questions 1-11 below:

1. A change in the payoff table, if it occurs, will only occur at the start of a new sequence. Circle one: True False

2. I will be matched with the same other participant in all rounds of a sequence. Circle one: True False

3. Suppose it is round 3 . The chance that the sequence continues on to round 4 is: Would your answer change if we replaced round 3 with round 13 and round 4 with round 14 ?

Circle one: Yes No

For the next four questions, assume payoff Table 1 is in effect.

4. If you choose $\mathrm{X}$ and the other participant matched with you chooses $\mathrm{Y}$, then your payoff is and the other participants payoff is .

5. If you choose $\mathrm{X}$ and the other participant matched with you chooses $\mathrm{X}$, then your payoff is and the other participants payoff is .

6. If you choose $\mathrm{Y}$ and the other participant matched with you chooses $\mathrm{Y}$, then your payoff is and the other participants payoff is .

7. If you choose $\mathrm{Y}$ and the other participant matched with you chooses $\mathrm{X}$, then your payoff is and the other participants payoff is .

For the next four questions, assume payoff Table 2 is in effect.

8. If you choose $\mathrm{X}$ and the other participant matched with you chooses $\mathrm{Y}$, then your payoff is and the other participants payoff is .

9. If you choose $\mathrm{X}$ and the other participant matched with you chooses $\mathrm{X}$, then your payoff is and the other participants payoff is .

10. If you choose $\mathrm{Y}$ and the other participant matched with you chooses $\mathrm{Y}$, then your payoff is and the other participants payoff is .

11. If you choose $\mathrm{Y}$ and the other participant matched with you chooses $\mathrm{X}$, then your payoff is and the other participants payoff is . 